\title{
A diamagnetic inequality for semigroup differences
}

\author{
By Dirk Hundertmark at Urbana and Barry Simon ${ }^{1)}$ at Pasadena
}

\begin{abstract}
The diamagnetic inequality for the magnetic Schrödinger semigroup is extended to the difference of the semigroups of magnetic Schrödinger operators with Neumann and Dirichlet boundary conditions on arbitrary open domains and rather general magnetic vector potentials $A$ and potentials $V$. In particular, this bound renders moot all the technical issues in the recent proofs of the independence of the boundary conditions for the integrated density of states for magnetic Schrödinger operators: Independence of the boundary conditions for the free case, that is, for vanishing potentials and vector potentials, immediately implies independence of the boundary conditions of the integrated density of states for a large class of magnetic Schrödinger operators.
\end{abstract}

\section{Introduction}

Let $H(A, V)=(-i \nabla-A)^{2}+V$ be the magnetic Schrödinger operator with electric potential $V$ and magnetic vector potential $A$. The diamagnetic inequality of Simon [45], [46] says that, under some rather general conditions on $A$ and $V$, the bound

$$
\left|\left(\mathrm{e}^{-t H(A, V)} f\right)(x)\right| \leqq\left(\mathrm{e}^{-t H(0, V)}|f|\right)(x)
$$

holds for all $t \geqq 0$ and almost all $x \in \mathbb{R}^{d}$. In some sense this inequality shows that the magnetic operator is dominated by the non-magnetic Schrödinger operator. The importance of this inequality was already noted in [2], [8]. Its strength lies in the fact that it is valid for rather arbitrary vector potentials $A$ and a large class of potentials $V$; see [19], [51] and [34] for further developments.

Our interest in the diamagnetic inequalities comes from the recent studies of the integrated density of states (IDS) of magnetic Schrödinger operators, especially the proofs of independence of the boundary conditions [14], [32], [21]. The integrated density of states is a fundamental quantity in the theory and applications of random Schrödinger operators [7], [23], [28], [37]. It is defined as follows: Let $\Lambda \subset \mathbb{R}^{d}$ be an open (and bounded) set, for

\footnotetext{
1) Supported in part by NSF grants DMS-9707661 and DMS-0140592.
} 
example, $\Lambda=\Lambda_{L}=(-L, L)^{d}$, and consider $H_{\Lambda}(A, V)$, the restriction of $H(A, V)$ to $L^{2}(\Lambda)$. Of course, one has to consider boundary conditions in order to get a self-adjoint operator. This is most conveniently done with the help of quadratic forms; see Section 2 . Since $\Lambda$ is bounded, the spectrum of $H_{\Lambda}(A, V)$ has a good chance of being discrete (at least with Dirichlet boundary conditions), in which case the eigenvalue distribution function

$$
N_{\Lambda}(\lambda)=\frac{1}{|\Lambda|} \#\left\{\text { eigenvalues of } H_{\Lambda}(A, V) \leqq \lambda\right\}
$$

is well-defined. The IDS is then given by the macroscopic, or infinite-volume, limit

$$
N(\lambda)=\lim _{\Lambda \rightarrow \mathbb{R}^{d}} N_{\Lambda}(\lambda)
$$

where $\Lambda \rightarrow \mathbb{R}^{d}$ is usually interpreted as $\Lambda=\Lambda_{L}=(-L, L)^{d}, L \rightarrow \infty$, or, more generally, in the sense of Fisher [16], [43]. We interpret the limit in (1.2) not in a pointwise sense, but as vague convergence of the corresponding measures. For example, (1.2) holds at all continuity points of $N$, in particular, for almost all $\lambda$. Basic questions are whether this limit exists at all, is independent of the chosen boundary conditions, and, in case the potential $V$ is random, that is, given by realizations of some random field, is independent of the realizations of the random potential.

For vanishing magnetic vector potentials, these questions had been solved some time ago [3], [7], [15], [23], [24], [33], [35], [36], [37]. For non-zero magnetic vector potentials, the existence and non-randomness of the IDS are well-known [5], [31], [56]. However, uniqueness has only recently been studied. Bounded potentials were considered in [32], extended to non-negative potentials and arbitrary vector potentials $A \in L_{\text {loc }}^{2}\left(\mathbb{R}^{d}, \mathbb{R}^{d}\right)$ in [14], and [21] used the method of [37] and the a priori input from [14] to extend this to some random potentials which are unbounded from below. All these results have somewhat technical and complicated proofs.

The main point of this paper is to show that independence of the boundary conditions of the IDS is a very natural property. In the free case, that is, for vanishing $A$ and $V$, it was already known to Weyl [60], see also [41], Chapter XIII.15, and we will show that the interacting system, for rather general vector potentials $A$ and potentials $V \geqq 0$, inherits this property from the free case. Thus it is a geometrical property of (the sequence of) the domains $\Lambda \rightarrow \mathbb{R}^{d}$.

To study the IDS for different boundary conditions, it is convenient, following Avron-Simon [3], to look at their Laplace transforms. Let $N_{\Lambda}^{\#}$ be the finitevolume IDS for \# $=N, D$, Neumann, respectively Dirichlet boundary conditions and $\mathscr{L}_{\Lambda}^{\#}(t)=\int \mathrm{e}^{-t \lambda} d N_{\Lambda}^{\#}(\lambda)$ be its Laplace transform. By the usual Dirichlet-Neumann bracketing,

$$
N_{\Lambda}^{D}(\lambda) \leqq N_{\Lambda}^{N}(\lambda)
$$

as long as $N_{\Lambda}^{N}$ is well-defined and consequently,

$$
0 \leqq \mathscr{L}_{\Lambda}^{D}(t) \leqq \mathscr{L}_{\Lambda}^{N}(t)
$$


Using the uniqueness theorem for Laplace transforms, it is enough to show that, for all fixed $t>0$,

$$
\lim _{\Lambda \rightarrow \mathbb{R}^{d}}\left(\mathscr{L}_{\Lambda}^{N}(t)-\mathscr{L}_{\Lambda}^{D}(t)\right)=0
$$

Let $\mathscr{L}_{\Lambda \text {,free }}^{\#}$ be the Laplace transforms of the free IDS, that is, for vanishing electric and vector potentials. From Weyl's result, we know that

$$
\mathscr{L}_{\Lambda, \text { free }}^{N}(t)-\mathscr{L}_{\Lambda, \text { free }}^{D}(t) \leqq \frac{C(t)}{|\Lambda|}(|\partial \Lambda|+1)
$$

for a large class of domains $\Lambda$. Of course, here one has to interpret $|\partial \Lambda|$ correctly, which is not a problem for domains with nice enough boundaries; see also Remark 1.5(iv) below, and one should keep in mind that, in our notation, the finite-volume IDS contains the factor $1 / \Lambda$. If one had a bound of the form

$$
\mathscr{L}_{\Lambda}^{N}(t)-\mathscr{L}_{\Lambda}^{D}(t) \leqq \mathscr{L}_{\Lambda, \text { free }}^{N}-\mathscr{L}_{\Lambda, \text { free }}^{D}
$$

then (1.5) would lead to the desired result (1.4), since the difference on the left-hand side is non-negative by Dirichlet-Neumann bracketing.

By the functional calculus, $\mathscr{L}_{\lambda}^{\#}(t)=\frac{1}{|\Lambda|} \operatorname{tr}\left[\mathrm{e}^{-t H_{\Lambda}^{\#}(A, V)}\right]$. Assume for the moment that the diamagnetic inequality (1.1) also holds for the operators restricted to $L^{2}(\Lambda)$, at least for Dirichlet and Neumann boundary conditions. Then some standard estimates on integral kernels [49] imply that

$$
\operatorname{tr}\left[\mathrm{e}^{-t H_{\Lambda}^{\#}(A, V)}\right] \leqq \operatorname{tr}\left[\mathrm{e}^{-t H_{\Lambda}^{\#}(0, V)}\right] \leqq \operatorname{tr}\left[\mathrm{e}^{-t H_{\Lambda}^{\#}(0,0)}\right]
$$

for $\#=D$ or $N$. The last inequality holds as long as $V \geqq 0$. In particular, $\mathscr{L}_{\Lambda}^{\#}(t) \leqq \mathscr{L}_{\Lambda \text {, free }}^{\#}(t)$. From this point of view, the inequality (1.6) looks rather strange, since it is in general not true that $a-b \leqq|a|-|b|$. However, as for the usual diamagnetic inequality (1.1), there is a simple probabilistic heuristic for the inequality (1.6): Let $b$ be the usual Brownian motion process in $\mathbb{R}^{d}$ and $T=\inf \left\{s \geqq 0: b_{s} \notin \Lambda\right\}$, the hitting time of the complement of $\Lambda$. The Feynman-Kac-Itô formula for the Dirichlet semigroup $\mathrm{e}^{-t H_{\Lambda}^{D}(A, V)}$ then reads

$$
\left(\mathrm{e}^{-t H_{\Lambda}^{D}(A, V)} f\right)(x)=\mathbb{E}^{x}\left[\mathrm{e}^{i I^{t}(b)-\int_{0}^{t} V\left(b_{s}\right) d s} \mathbf{1}_{\{T<t\}}(b) f\left(b_{t}\right)\right] .
$$

Here $\mathbb{E}^{x}$ stands for integrating with respect to Brownian motion starting at $x, \mathbf{1}_{A}$ is the indicator function of the set $A$, and $I^{t}$ is the line integral of $A$ along Brownian paths. Since Brownian paths are not of finite variation, this needs some interpretation; see, e.g., [49], Chapters 14 and 15, [42], Chapter 6, and [6]. Usually one also has to impose some additional conditions on the divergence of $A$ for this probabilistic approach. In any case, since both $I^{t}(b)$ and $\int_{0}^{t} V\left(b_{s}\right) d s$ are real-valued random variables, the Feynman-Kac-Itô formula together with the triangle inequality immediately implies (1.1). So from a probabilistic point of view, the diamagnetic inequality is, at least for Dirichlet boundary conditions, just 
the triangle inequality for integrals. This point of view of the diamagnetic inequalities goes back to Nelson; see [49], Chapter 15.

Assume for the moment that a Feynman-Kac-Itô type formula also holds for the Neumann semigroup, that is,

$$
\left(\mathrm{e}^{-t H_{\Lambda}^{N}(A, V)} f\right)(x)=\tilde{\mathbb{E}}^{x}\left[\mathrm{e}^{i I^{t}(\tilde{b})-\int_{0}^{t} V\left(\tilde{b}_{s}\right) d s} f\left(\tilde{b}_{t}\right)\right],
$$

where $\tilde{b}$ is reflected Brownian motion. The magnetic phase $I^{t}$ and $\int_{0}^{t} V\left(b_{s}\right) d s$ depend only locally on the paths. Moreover, one has $b_{s}=\tilde{b}_{s}$ for $s \leqq t$ and paths in $\{T \leqq t\}$, since, at least morally, reflected Brownian motion up to the stopping time $T$ has not reached the boundary and hence is not reflected, yet. Accordingly, we can extend the integration in (1.7) to all reflected paths $\tilde{b}$ and, subtracting it from (1.8), one gets

$$
\begin{aligned}
\left|\left(\mathrm{e}^{-t H_{\Lambda}^{N}(A, V)}-\mathrm{e}^{-t H_{\Lambda}^{D}(A, V)}\right) f(x)\right| & =\left|\tilde{\mathbb{E}}_{x}\left[\mathrm{e}^{i I^{t}(\tilde{b})-\int_{0}^{t} V\left(\tilde{b}_{s}\right) d s}\left(1-\mathbf{1}_{\{T<t\}}(\tilde{b})\right) f\left(\tilde{b}_{t}\right)\right]\right| \\
& \leqq \tilde{\mathbb{E}}_{x}\left[\mathrm{e}^{-\int_{0}^{t} V\left(\tilde{b}_{s}\right) d s}\left(1-\mathbf{1}_{\{T<t\}}(\tilde{b})\right)\left|f\left(\tilde{b}_{t}\right)\right|\right] \\
& =\left(\mathrm{e}^{-t H_{\Lambda}^{N}(0, V)}-\mathrm{e}^{-t H_{\Lambda}^{D}(0, V)}\right)|f|(x),
\end{aligned}
$$

since $1-\mathbf{1}_{\{T<t\}}=\mathbf{1}_{\{T \geqq t\}} \geqq 0$. Arguing the same way, one also sees

$$
\left|\left(\mathrm{e}^{-t H_{\Lambda}^{N}(0, V)}-\mathrm{e}^{-t H_{\Lambda}^{D}(0, V)}\right) f(x)\right| \leqq\left(\mathrm{e}^{-t H_{\Lambda}^{N}(0,0)}-\mathrm{e}^{-t H_{\Lambda}^{D}(0,0)}\right)|f|(x)
$$

for all non-negative potentials $V$. Taking traces, (1.9) and (1.10) imply (1.6), at least on a formal level. Hence, independence of the boundary conditions of the integrated states should follow from the free case.

The catch in the above argument is that we do not know whether a Feynman-Kac-Itô type formula holds for the Neumann case. Although this is conceivably the case, it will pose strong restrictions on the domain $\Lambda$. It is well-known that Neumann boundary conditions are a tricky business and even the free Neumann operator on bounded domains can have some surprising spectral properties, like non-trivial essential spectrum or even nontrivial "scattering" asymptotics [12], [18], [52]. In particular, reflected Brownian motion will exist only for nice enough domains [55], [61], [62]. Moreover, some restrictions on $\Lambda$ will already have to be imposed in order that the finite-volume $\operatorname{IDS}, N_{\Lambda}^{N}$, is well defined, see Remark 1.5(ii), but we do not want to impose these restrictions from the beginning in the proof of the diamagnetic inequalities. So instead, we use the probabilistic heuristic as a guiding principle for the right kind of inequality to be proven, that is, (1.9) and (1.10), and will give an analytic proof for them. Moreover, it is conceivable that a probabilistic approach will also need some regularity assumptions on $A$ near the boundary of $\Lambda$, whereas the analytic approach we propose will only need $\Lambda$ open and $A \in L_{\text {loc }}^{2}\left(\Lambda\right.$, $\left.\mathbb{R}^{d}\right)$, a condition which is already very convenient for the definition of the operators $H_{\Lambda}^{N}(A, V)$ and $H_{\Lambda}^{D}(A, V)$ via quadratic forms, see Section 2, and thus poses no further restriction on the class of vector potentials to be considered. As a bonus, we obtain a new proof of the normal diamagnetic inequalities which is "form theoretic" rather than the operator theoretic proof of Simon [46], [51]. 
The main result of this paper is given by the following two theorems:

Theorem 1.1. Let $\Lambda \subset \mathbb{R}^{d}$ be open, $A \in L_{\text {loc }}^{2}\left(\Lambda, \mathbb{R}^{d}\right)$, and $V=V_{+}-V_{-}$with $V_{ \pm} \geqq 0$ and $V_{+} \in L_{\mathrm{loc}}^{1}(\Lambda), V_{-}$relatively form bounded w.r.t. $H_{\Lambda}^{N}(0,0)$. Then, for all $f \in L^{2}(\Lambda)$ and almost all $x \in \Lambda$,

$$
\left|\mathrm{e}^{-t H_{\Lambda}^{N}(A, V)} f(x)\right| \leqq \mathrm{e}^{-t H_{\Lambda}^{N}(0, V)}|f|(x) \leqq \mathrm{e}^{-t H_{\Lambda}^{N}\left(0,-V_{-}\right)}|f|(x) .
$$

Remarks 1.2. (i) Given the discussion above, this result is certainly to be expected, but, maybe somewhat surprisingly, no regularity assumptions on $A$ and $V_{+}$are made close to the boundary of $\Lambda$. Also, since $\Lambda$ is an arbitrary open set, its boundary can be quite wild. Following the approach in [50], a weaker version of the first inequality is proven in [20] under the condition that $A$ and the positive part of the potential $V_{+}$are restrictions of a vector potential in $L_{\text {loc }}^{2}\left(\mathbb{R}^{d}, \mathbb{R}^{d}\right)$ and a potential in $L_{\text {loc }}^{1}\left(\mathbb{R}^{d}\right)$.

(ii) Choosing for $f$ an approximate delta-function, Theorem 1.1 implies the bound $\left|\mathrm{e}^{-t H_{\Lambda}^{N}(A, V)}(x, y)\right| \leqq \mathrm{e}^{-t H_{\Lambda}^{N}(0, V)}(x, y)$ for almost all $(x, y) \in \Lambda \times \Lambda$ for the integral kernel of the Neumann semigroup (as long as it exists).

(iii) Of course, the analogous result for Dirichlet boundary conditions also holds.

The second theorem shows that the probabilistic heuristic, suggesting a diamagnetic inequality for the difference of the Neumann and Dirichlet semigroup, is, indeed, correct.

Theorem 1.3. Let $\Lambda \subset \mathbb{R}^{d}$ be open, $A \in L_{\text {loc }}^{2}\left(\Lambda, \mathbb{R}^{d}\right)$, and $V=V_{+}-V_{-}$with $V_{ \pm} \geqq 0$ and $V_{+} \in L_{\text {loc }}^{1}(\Lambda), V_{-}$relatively form bounded w.r.t. $H_{\Lambda}^{N}(0,0)$. Then

$$
\begin{aligned}
\left|\left(\mathrm{e}^{-t H_{\Lambda}^{N}(A, V)}-\mathrm{e}^{-t H_{\Lambda}^{D}(A, V)}\right) f(x)\right| & \leqq\left(\mathrm{e}^{-t H_{\Lambda}^{N}(0, V)}-\mathrm{e}^{-t H_{\Lambda}^{D}(0, V)}\right)|f|(x) \\
& \leqq\left(\mathrm{e}^{-t H_{\Lambda}^{N}\left(0,-V_{-}\right)}-\mathrm{e}^{-t H_{\Lambda}^{D}\left(0,-V_{-}\right)}\right)|f|(x)
\end{aligned}
$$

for all $f \in L^{2}(\Lambda)$ and almost all $x \in \Lambda$.

Recall that an open set $\Lambda$ has the extension property if, with $W^{1, p}(\Lambda)$ denoting the usual Sobolev space, there exists a bounded extension operator $E: W^{1, p}(\Lambda) \rightarrow W^{1, p}\left(\mathbb{R}^{d}\right)$ for all $1 \leqq p<\infty$. If $\Lambda$ has the extension property, then it is known that the free Neumann semigroup $\mathrm{e}^{-t H_{\Lambda}^{N}(0,0)}$ is an integral operator, see [11], Theorem 2.4.4. If, moreover, $\Lambda$ is also bounded, then $\mathrm{e}^{-t H_{\Lambda}^{N}(0,0)}$ is a trace class operator for all $t>0$; see Remarks 1.5(ii) and 1.5(iii) below. An immediate corollary of the two theorems above is given by

Corollary 1.4. Let $\Lambda$ be a bounded set having the extension property.

(i) For any $t>0, \mathrm{e}^{-t H_{\Lambda}^{N}(A, V)}$ is a trace class operator for arbitrary vector potentials $A \in L_{\mathrm{loc}}^{2}\left(\Lambda, \mathbb{R}^{d}\right)$, and potentials $V=V_{+}-V_{-}$with $V_{ \pm} \geqq 0, V_{+} \in L_{\mathrm{loc}}^{1}(\Lambda)$, and $V_{-}$form small with respect to $H_{\Lambda}^{N}(0,0)$. Moreover,

$$
\operatorname{tr}_{L^{2}(\Lambda)}\left[\mathrm{e}^{-t H_{\Lambda}^{N}(A, V)}\right] \leqq \operatorname{tr}_{L^{2}(\Lambda)}\left[\mathrm{e}^{-t H_{\Lambda}^{N}(0, V)}\right] \leqq \operatorname{tr}_{L^{2}(\Lambda)}\left[\mathrm{e}^{-t H_{\Lambda}^{N}\left(0,-V_{-}\right)}\right]
$$

for all $t>0$. 
(ii) The bounds

$$
\begin{aligned}
0 \leqq \operatorname{tr}_{L^{2}(\Lambda)}\left[\mathrm{e}^{-t H_{\Lambda}^{N}(A, V)}-\mathrm{e}^{-t H_{\Lambda}^{D}(A, V)}\right] & \leqq \operatorname{tr}_{L^{2}(\Lambda)}\left[\mathrm{e}^{-t H_{\Lambda}^{N}(0, V)}-\mathrm{e}^{-t H_{\Lambda}^{D}(0, V)}\right] \\
& \leqq \operatorname{tr}_{L^{2}(\Lambda)}\left[\mathrm{e}^{-t H_{\Lambda}^{N}\left(0,-V_{-}\right)}-\mathrm{e}^{-t H_{\Lambda}^{D}\left(0,-V_{-}\right)}\right]
\end{aligned}
$$

hold for all $A \in L_{\mathrm{loc}}^{2}\left(\Lambda, \mathbb{R}^{d}\right)$.

The proof of this corollary is given at the end of Section 3.

Remarks 1.5. (i) For the free Dirichlet semigroup $\mathrm{e}^{-t H_{\Lambda}^{D}(0,0)}$ to be trace class for $t>0$, it is enough that $\Lambda$ is bounded, see [41], Theorem XIII.76. The analog of Corollary 1.4(i) holds for the Dirichlet semigroup assuming only boundedness of $\Lambda$.

(ii) Any bounded convex domain and every bounded domain with a piecewise smooth boundary has the extension property. In general, a domain which is "minimally smooth" will have the extension property; see [44], page 189, for the precise conditions. The discussion in [11], Chapters 1 and 2, also shows that if $\Lambda$ is bounded and has the extension property, then the spectrum of $H_{\Lambda}^{N}(0,0)$ is discrete, since $H_{\Lambda}^{N}(0,0)$ can be compared with the Dirichlet Laplacian on a large enough ball containing $\Lambda$. For that reason, one knows also that the eigenvalues of $H_{\Lambda}^{N}(0,0)$ go to infinity fast enough such that $\mathrm{e}^{-t H_{\Lambda}^{N}(0,0)}$ is a trace class operator. In particular, for these domains, the spectrum of $H_{\Lambda}^{N}(0,0)$ and of $H_{\Lambda}^{N}(A, V)$ is discrete. Thus the finite-volume IDS for Neumann boundary conditions, $N_{\Lambda}^{N}$, is welldefined.

(iii) By the semigroup property, $\mathrm{e}^{-t H_{\Lambda}^{N}(0,0)}$ is a trace class operator for all $t>0$ as soon as it is in some von Neumann-Schatten ideal $\mathscr{I}_{p}$ for all small enough $t>0$ and some finite $p$. It seems conceivable that, by mimicking the rooms-and-passages construction in [18], one could construct a bounded open set $\Lambda$, necessarily not having the extension property, such that for some $t_{0}>0, \mathrm{e}^{-t_{0} H_{\Lambda}^{N}(0,0)}$ is compact but not in any von NeumannSchatten ideal for finite $p$.

(iv) Corollary 1.4 shows that for non-negative (or, more generally, bounded below) potentials and arbitrary vector potentials, the IDS is independent of the boundary conditions as soon as this is true for the free case. If the sequence of domains is given by boxes, $\Lambda=\left\{-L_{j} / 2<x_{j}<L_{j} / 2, j=1, \ldots, d\right\}$, this again can be seen rather naturally by probabilistic methods. With the help of the method of images, one can give an explicit expression for $\mathrm{e}^{-t H_{\Lambda}^{N}(0,0)}$ in terms of a Feynman-Kac formula, see [4], Example 6.3.11, and [24]. Using this, one can easily show that for any $\varepsilon>0$ and with $\partial \Lambda_{\varepsilon}=\left\{x \in \Lambda \mid \operatorname{dist}\left(x, \Lambda^{c}\right) \leqq \varepsilon\right\}$,

$$
\operatorname{tr}\left[\mathrm{e}^{-t H_{\Lambda}^{N}(0,0)}-\mathrm{e}^{-t H_{\Lambda}^{D}(0,0)}\right] \leqq C_{t}\left(\left|\partial \Lambda_{\varepsilon}\right|+\mathrm{e}^{-\varepsilon^{2} /(2 t)}|\Lambda|\right),
$$

as was already noticed in [35]. Thus a suitably modified version of (1.5) holds. Hence, for sequences $\Lambda \rightarrow \mathbb{R}^{d}$ of boxes (for which one always has $\left|\partial \Lambda_{\varepsilon}\right| /|\Lambda| \rightarrow 0$ for fixed $\varepsilon>0$ ), the IDS is independent of the boundary conditions used in its definition for arbitrary vector potentials $A$ and non-negative potentials $V$.

(v) Using the methods of [15], [24] or [37], one can extend this result to certain not 
necessarily non-negative potentials $V$. The most general result in this direction is in [21], which uses the a priori information that the IDS is independent of the boundary conditions for non-negative potentials, or equivalently, potentials which are bounded from below. In particular, it is shown that for $\mathbb{R}^{d}$-ergodic random potentials, the IDS is independent of the boundary conditions as soon as $\mathbb{E}\left[\left(V_{\omega}(0)\right)_{-}^{p}\right]<\infty$ for some $p>d+1$. A straightforward modification of the result in [25], see also [23], Theorem 1 in Section 5, shows that for differentiable vector potentials $A$, the random magnetic Schrödinger operator $(-i \nabla+A)^{2}+V_{\omega}$ is almost surely self-adjoint on $\mathscr{C}_{0}^{\infty}\left(\mathbb{R}^{d}\right)$ as long as $p>d$. This leaves open the question of uniqueness of the IDS for $d<p \leqq d+1$. We are convinced that uniqueness holds as soon as one has essential self-adjointness, that is, one should have uniqueness for $p>d$.

The organization of the paper is as follows: In Section 2 we give the construction of the magnetic Schrödinger operator on arbitrary open domains with Neumann and Dirichlet boundary conditions and gather some technical tools. Theorems 1.1 and 1.3 are proven in Section 3. In the probabilistic heuristics given above, the main observation was that the diamagnetic inequality for the difference of semigroups follows from the Feynman-Kac-Itô representation (1.8). In our analytic proof, this is replaced by the diamagnetic inequality for the Neumann semigroup: Modulo some approximation arguments, Theorem 1.3 is a corollary of Theorem 1.1; we first prove a result similar to that in Theorem 1.3 for the difference of two Neumann semigroups whose generators differ by a positive potential, see Lemma 3.5, and then use an approximation argument to recover Dirichlet from Neumann boundary conditions. The main tool for the proof of Theorem 1.1 is given in Lemma 3.1. For the convenience of the reader, we present the approximation theorem needed in the proof of Theorem 1.3 in the appendix.

\section{Some preliminaries}

We begin with some general preliminaries. Even without magnetic vector potentials, restricting the Laplacian to an open subset poses the problem of introducing the right boundary conditions for which the restriction is realized by a self-adjoint operator. This is most conveniently done with the help of quadratic forms. It turns out that introducing a magnetic vector potential poses no real difficulty, except some notational effort.

Let $\Lambda \subset \mathbb{R}^{d}$ be an open set. For $1 \leqq p \leqq \infty$, we let $L^{p}(\Lambda)=L^{p}(\Lambda, \mathbb{C})$ be the usual Lebesgue spaces of complex-valued functions whose $p^{\text {th }}$ power is integrable, and $L_{\text {loc }}^{p}(\Lambda)$ be the space of (measurable) functions $f: \Lambda \rightarrow \mathbb{C}$ for which $f \mathbf{1}_{K} \in L^{p}(\Lambda)$ for all compact subsets $K \subset \Lambda$. Here $\mathbf{1}_{K}$ denotes the indicator function of the set $K$. Furthermore, for $\mathbb{K}$ being either $\mathbb{R}$ or $\mathbb{C}$, the spaces $L^{p}\left(\Lambda, \mathbb{K}^{d}\right)$, respectively $L_{\text {loc }}^{p}\left(\Lambda, \mathbb{K}^{d}\right)$, are the Lebesgue spaces of functions $f: \Lambda \rightarrow \mathbb{K}^{d}$ whose coordinate functions $f_{j}: \Lambda \rightarrow \mathbb{K}$ are in $L^{p}(\Lambda)$, respectively $L_{\text {loc }}^{p}(\Lambda)$. For non-negative functions $f$ and $g$, we write $f \lesssim g$ if, for some constant $C>0$, one has $f \leqq C g$.

We write $\mathscr{C}_{0}^{\infty}(\Lambda)$ for the infinitely often differentiable functions with compact support in $\Lambda$. Like the usual gradient, for a magnetic vector potential $A \in L_{\text {loc }}^{2}(\Lambda)$, the magnetic gradient $D_{A}=\nabla-i A$ is a closable operator on $\mathscr{C}_{0}^{\infty}(\Lambda)$; more precisely, its components $D_{A, j}=\frac{\partial}{\partial x_{j}}-i A_{j}$ are closable since they are anti-symmetric operators with respect to the 
standard scalar product $\langle f, g\rangle=\int_{\Lambda} \bar{f} g d x$ on $L^{2}(\Lambda)$, that is, $\left\langle\varphi, D_{A, j} \psi\right\rangle=\left\langle-D_{A, j} \varphi, \psi\right\rangle$ for all $\varphi, \psi \in \mathscr{D}\left(D_{A}\right)$ and $j=1, \ldots, d$. By slight abuse of notation, we will use $D_{A, j}$ to denote this closure. The magnetic Sobolev space $H_{A, 0}^{1}(\Lambda)\left(=W_{A, 0}^{1,2}(\Lambda)\right)$ is then given by $H_{A, 0}^{1}(\Lambda):=\bigcap_{d}^{d} \mathscr{D}\left(D_{A, j}\right)$, which, for $A=0$, reduces to the well-known Sobolev space $H_{0}^{1}(\Lambda)=W_{0}^{1,2}(\Lambda)$. The operator $H_{\Lambda}^{D}(A, 0)$ is defined as the unique operator associated with the closed, non-negative symmetric quadratic form

$$
h_{\Lambda}^{D}(A, 0)(\varphi, \psi):=\sum_{j=1}^{d}\left\langle D_{A, j} \varphi, D_{A, j} \psi\right\rangle
$$

with form domain $\mathscr{D}\left(h_{\Lambda}^{D}(A, 0)\right):=H_{A, 0}^{1}(\Lambda)$. This gives the well-known construction for the Dirichlet Laplacian $H_{\Lambda}^{D}(0,0)=-\Delta_{\Lambda}^{D}$ for vanishing magnetic vector potentials.

For the Neumann boundary conditions, we need the maximal magnetic gradient, which is the magnetic analog of the maximal gradient. Since $D_{A, j}$ is an anti-symmetric operator, a natural closed extension is given by the negative of its adjoint, $D_{A, \max , j}=-\left(D_{A, j}\right)^{*}$, for $j=1, \ldots, d$. That is,

$$
\int_{\Lambda} \bar{\varphi} D_{A, \max , j} f d x:=-\int_{\Lambda} \overline{D_{A, j} \varphi} f d x
$$

for all $\varphi \in \mathscr{C}_{0}^{\infty}(\Lambda) \subset \mathscr{D}\left(D_{A}\right)$. The domain of $D_{A \text {, max }}$ is given by

$$
H_{A}^{1}(\Lambda) \equiv \mathscr{D}\left(D_{A, \max }\right):=\bigcap_{j=1}^{d} \mathscr{D}\left(D_{A, \max , j}\right)
$$

with

$$
\begin{aligned}
& \mathscr{D}\left(D_{A, \max , j}\right)=\mathscr{D}\left(D_{A, j}^{*}\right)=\left\{f \in L^{2}(\Lambda) \mid \text { there exists } \eta_{f} \in L^{2}(\Lambda)\right. \text { such that } \\
&\left.\left\langle-D_{A, j} \varphi, f\right\rangle=\left\langle\varphi, \eta_{f}\right\rangle \text { for all } \varphi \in \mathscr{C}_{0}^{\infty}(\Lambda)\right\} \\
&=\left\{f \in L^{2}(\Lambda) \mid \partial_{j} f \in L_{\mathrm{loc}}^{1}(\Lambda), A_{j} f \in L_{\mathrm{loc}}^{1}(\Lambda)\right. \\
&\text { with } \left.\partial_{j} f-i A_{j} f \in L^{2}(\Lambda)\right\},
\end{aligned}
$$

where the partial derivative $\partial_{j} f$ is in the weak sense. $D_{0, \max }$ is the well-known maximal (or distributional) gradient $\nabla_{\max }$. Since $A \in L_{\text {loc }}^{2}\left(\Lambda, \mathbb{R}^{d}\right)$, by assumption, we have Af $\in L_{\mathrm{loc}}^{1}\left(\Lambda, \mathbb{C}^{d}\right)$ for all $f \in L^{2}(\Lambda)$. Thus,

$$
H_{A}^{1}(\Lambda)=\left\{f \in L^{2}(\Lambda) \cap W_{\mathrm{loc}}^{1,1}(\Lambda) \mid D_{A, \max } f \in L^{2}\left(\Lambda, \mathbb{C}^{d}\right)\right\} \subset L^{2}(\Lambda) \cap W_{\mathrm{loc}}^{1,1}(\Lambda) .
$$

Here $W_{\text {loc }}^{1,1}(\Lambda)=\left\{f \in L_{\text {loc }}^{1}(\Lambda) \mid \nabla_{\max } f \in L_{\text {loc }}^{1}(\Lambda)\right\}$. Thus, in general, one only knows that $\nabla_{\max } f \in L_{\text {loc }}^{1}(\Lambda)$ for $f \in \mathscr{D}\left(D_{A, \max }\right)$, which is the main reason for some of the technical difficulties with magnetic Schrödinger operators.

The Neumann quadratic form is given by the closed, non-negative, symmetric form 


$$
h_{\Lambda}^{N}(A, 0)(\varphi, \psi):=\sum_{j=1}^{d}\left\langle D_{A, \max , j}(\varphi), D_{A, \max , j}(\psi)\right\rangle
$$

with domain $\mathscr{D}\left(h_{\Lambda}^{N}(A, 0)\right):=H_{A}^{1}(\Lambda)$. Again, it uniquely defines the Neumann magnetic Laplacian $H_{\Lambda}^{N}(A, 0)$, which reduces to $-\Delta_{\Lambda}^{N}$ for $A=0$.

Remark 2.1. Let \# be a boundary condition which leads to a quadratic form which is sandwiched between the Dirichlet and Neumann forms, that is, for which $\mathscr{D}\left(h_{\Lambda}^{D}(A, 0)\right) \subset \mathscr{D}\left(h_{\Lambda}^{\#}(A, 0)\right) \subset \mathscr{D}\left(h_{\Lambda}^{N}(A, 0)\right)$ and $h_{\Lambda}^{N}(A, 0)[\varphi, \varphi] \leqq h_{\Lambda}^{\#}(A, 0)[\varphi, \varphi]$ for all $\varphi \in \mathscr{D}\left(h_{\Lambda}^{\#}[A, 0]\right)$ together with $h_{\Lambda}^{\#}(A, 0)[\varphi, \varphi] \leqq h_{\Lambda}^{D}(A, 0)[\varphi, \varphi]$ for all $\varphi \in \mathscr{D}\left(h_{\Lambda}^{D}[A, 0]\right)$ holds. For such a boundary condition, the min-max principle gives the bound $N_{\Lambda}^{D}(\lambda) \leqq N_{\Lambda}^{\#}(\lambda) \leqq N_{\Lambda}^{N}(\lambda)$ for the finite volume IDS $N^{\#}$ and consequently $\mathscr{L}_{\Lambda}^{D}(t) \leqq \mathscr{L}_{\Lambda}^{\#}(t) \leqq \mathscr{L}_{\Lambda}^{N}(t)$ for the Laplace transforms. Thus, for this type of boundary conditions, the finite-volume IDS $N_{\Lambda}^{\#}$ is well-defined as soon as this is the case for Neumann boundary conditions and its infinite volume limit will not depend on the chosen boundary condition as soon as the infinite volume limits constructed with the help of the Neumann and Dirichlet boundary conditions are the same. However, one should note that there are self-adjoint extensions of $(-i \nabla+A)^{2}$ on finite domains, which have associated quadratic forms not comparable to the Dirichlet or Neumann form even for vanishing magnetic vector potential.

To include an electric potential $V \in L_{\text {loc }}^{1}(\Lambda)$, first assume that it is non-negative. Then, by setting $v[\varphi, \psi]=\langle\sqrt{V} \varphi, \sqrt{V} \psi\rangle, V$ can be seen as a closed quadratic form with domain $\mathscr{Z}(V)=\mathscr{D}(\sqrt{V})$ which includes $\mathscr{C}_{0}^{\infty}(\Lambda)$. Hence, $\mathscr{Z}(V) \cap \mathscr{D}\left(h_{\Lambda}^{\#}(A, 0)\right)$ is dense in $L^{2}(\Lambda)$ and

$$
h_{\Lambda}^{\#}[A, V](\varphi, \psi):=h_{\Lambda}^{\#}[A, 0](\varphi, \psi)+\langle\sqrt{V} \varphi, \sqrt{V} \psi\rangle
$$

on $\mathscr{D}\left(h_{\Lambda}^{\#}[A, V]\right):=\mathscr{D}\left(h_{\Lambda}^{\#}[A, 0]\right) \cap \mathscr{D}(\sqrt{V})$ are closed, densely defined quadratic forms, generating the magnetic Schrödinger operators $H_{\Lambda}^{\#}(A, V)$ with Dirichlet, \# $=D$, or Neumann, \# $=N$, boundary conditions.

If the potential is not necessarily non-negative, write $V$ as $V=V_{+}-V_{-}$with $V_{ \pm}:=\sup (0, \pm V)$. Assuming $V_{+} \in L_{\mathrm{loc}}^{1}(\Lambda)$ and $V_{-}$form small with respect to $h_{\Lambda}^{\#}\left[A, V_{+}\right]$, $\left\langle\varphi, V_{-} \varphi\right\rangle \leqq a_{\#} h^{\#}\left[A, V_{+}\right](\varphi, \varphi)+b\langle\varphi, \varphi\rangle$ for some $0 \leqq a_{\#}<1$ and $0 \leqq b<\infty$, the KLMN theorem, see, e.g., [40], shows that the form sum

$$
h_{\Lambda}^{\#}[A, V](\varphi, \psi):=h^{\#}\left[A, V_{+}\right](\varphi, \psi)-\left\langle\sqrt{V_{-}} \varphi, \sqrt{V_{-}} \psi\right\rangle
$$

defines a closed quadratic form which is bounded from below. It again uniquely defines a self-adjoint magnetic Schrödinger operator $H_{\Lambda}^{\#}(A, V)$.

Remarks 2.2. (i) Since $h_{\Lambda}^{D}\left(A, V_{+}\right)$is the restriction of $h_{\Lambda}^{N}\left(A, V_{+}\right)$to $H_{A, 0}^{1}(\Lambda) \cap \mathscr{Z}\left(V_{+}\right)$, any potential $V_{-}$which is form small with respect to the Neumann form is also form small with respect to $h_{\Lambda}^{D}\left[A, V_{+}\right]$.

(ii) As a consequence of the diamagnetic inequality in Theorem 3.3, any potential which is form small with respect to $h^{\#}\left[0, V_{+}\right]$with relative bound $a_{\#}$, for \# $=D$ or $N$, is 
also form small with respect to $h^{\#}\left[A, V_{+}\right]$with relative bound $\tilde{a}_{\#} \leqq a_{\#}$ for any magnetic vector potential $A \in L_{\mathrm{loc}}^{2}\left(\Lambda, \mathbb{R}^{d}\right)$.

(iii) The conditions we impose on the potential are not the weakest possible, but are general enough to cover all cases of interest for the applications we have in mind. It is possible to study a larger class of positive perturbations. Without magnetic vector potentials, this has been extensively studied in [53], [54], [57], [58]. For example, the condition $V_{+} \in L_{\mathrm{loc}}^{1}(\Lambda)$ can be replaced by the assumption of regularity, that is, $\mathscr{2}\left(V_{+}\right) \cap \mathscr{D}\left(h_{\Lambda}^{\#}(A, 0)\right)$ is dense in $L^{2}(\Lambda)$. This allows for somewhat strong local singularities in $V_{+}$, [54]. More general magnetic vector potentials, relaxing the condition $A^{2} \in L_{\mathrm{loc}}^{1}\left(\mathbb{R}^{d}\right)$ somewhat in the spirit of [57], [58], were discussed in [29].

Our main tool for the diamagnetic inequality is Lemma 3.1 below. We collect some preparatory technical tools first.

Lemma 2.3. (i) If $f, g \in W_{\mathrm{loc}}^{1,1}(\Lambda)$ with $f \nabla_{\max } g$ and $g \nabla_{\max } f \in L_{\mathrm{loc}}^{1}(\Lambda)$, then $f g \in W_{\mathrm{loc}}^{1,1}(\Lambda)$ and the product rule

$$
\nabla_{\max }(f g)=f \nabla_{\max } g+g \nabla_{\max } f
$$

holds.

(ii) If $u \in W_{\mathrm{loc}}^{1,1}(\Lambda)$ is real valued with $u \geqq \varepsilon>0$, then $\frac{1}{u} \in W_{\mathrm{loc}}^{1,1}(\Lambda)$ and

$$
\nabla_{\max } \frac{1}{u}=-\frac{\nabla_{\max } u}{u^{2}}
$$

Remark 2.4. Part (i) of this lemma already appeared in [30], Hilfssatz 14.1. The usual formulation of this result requires $f \in W_{\mathrm{loc}}^{1, p}(\Lambda)$ and $g \in W_{\mathrm{loc}}^{1, p^{\prime}}(\Lambda)$ with $1 / p+1 / p^{\prime}=1$. It will be very convenient to have it formulated in this generality for the proof of Lemma 2.5 below.

Proof. Since the conclusions of the lemma are local, it is enough to fix some compact subset $K \subset \Lambda$ and to prove the result for almost all $x \in K$. Let $j_{\delta}$ be an approximate delta function with $\operatorname{supp}\left(j_{\delta}\right) \in B_{\delta}=\{\|x\|<\delta\}$. We extend all functions to $\mathbb{R}^{d}$ by setting them to zero on $\Lambda^{c}$. Thus, for any $f \in L_{\text {loc }}^{1}(\Lambda)$, the convolution $f_{\delta}=j_{\delta} * f$ is well-defined. Moreover, for small enough $\delta, u_{\delta}(x) \geqq \varepsilon$ for all $x \in K$.

To prove part (ii), note that for the smoothed function $u_{\delta}$, the conclusion of the lemma is immediate. Now take $\delta \rightarrow 0$ along a subsequence chosen such that the individual terms converge almost everywhere and use the definition of the weak derivative.

To prove part (i), first assume that $f, g$ are bounded. Then $\left\|f_{\delta}\right\|_{\infty} \leqq\|f\|_{\infty}$ and the same for $g$. Again choose a subsequence $\delta_{n} \rightarrow 0$ such that individual terms converge almost everywhere in $\Lambda$. Writing $\nabla$ for $\nabla_{\max }$ and using dominated convergence, $\left\|f_{\delta_{n}} \nabla g_{\delta_{n}}-f \nabla g\right\|_{L^{1}(\Lambda)} \leqq\|f\|_{\infty}\left\|\nabla g_{\delta_{n}}-\nabla g\right\|+\left\|\left(f_{\delta_{n}}-f\right) \nabla g\right\|_{L^{1}(\Lambda)} \rightarrow 0$ as $\delta_{n} \rightarrow 0$. Similarly, $g_{\delta_{n}} \nabla f_{\delta_{n}} \rightarrow g \nabla f$ and $f_{\delta_{n}} g_{\delta_{n}} \rightarrow f g$ as $\delta_{n} \rightarrow 0$. Since $\nabla\left(f_{\delta} g_{\delta}\right)=g_{\delta} \nabla f_{\delta}+f_{\delta} \nabla g_{\delta}$, by the usual product rule, we see that $f g \in W^{1,1}(\Lambda)$ and $\nabla(f g)=g \nabla f+f \nabla g$ in the limit $\delta_{n} \rightarrow 0$. Now 
assume $f$ and $g$ real-valued, but not necessarily bounded. Put $f_{n}=\mathbf{1}_{\{|f| \leqq n\}} f$ and similarly for $g$. By the usual arguments in the theory of Sobolev spaces, see, e.g., [17], Lemma 7.6, we have $f_{n}, g_{n} \in W^{1,1}(\Lambda)$ with $\nabla f_{n}=\mathbf{1}_{\{|f| \leqq n\}} \nabla f$ and $\nabla g_{n}=\mathbf{1}_{\{|g| \leqq n\}} \nabla g$. Thus $f_{n} g_{n} \in W^{1,1}(\Lambda)$ with $\nabla\left(f_{n} g_{n}\right)=g_{n} \mathbf{1}_{\{|f| \leqq n\}} \nabla f+f_{n} \mathbf{1}_{\{|g| \leqq n\}} \nabla g$. Letting $n \rightarrow \infty$, using dominated convergence, gives the claim. For $f$ and $g$ complex-valued the result follows by writing them as a sum of their real and imaginary parts.

Following Kato [22], for each $\varepsilon>0$ and $u: \Lambda \rightarrow \mathbb{C}$ measurable, let

$$
u_{\varepsilon}:=\sqrt{|u|^{2}+\varepsilon^{2}} \geqq \varepsilon \quad \text { and } \quad s_{\varepsilon}:=u /|u|_{\varepsilon} .
$$

Furthermore, set $s(x):=u(x) /|u(x)|$ where $u(x) \neq 0$ and $s(x)=0$ whenever $u(x)=0$. Then $s_{\varepsilon}(x) \rightarrow s(x)$ for all $x$ as $\varepsilon \rightarrow 0$.

Lemma 2.5. Let $u \in W_{\mathrm{loc}}^{1,1}(\Lambda)$. Then:

(i) $|u|, u_{\varepsilon} \in W_{\text {loc }}^{1,1}(\Lambda)$ for all $\varepsilon>0$ with $\nabla_{\max } u_{\varepsilon}=\operatorname{Re}\left(\overline{s_{\varepsilon}} \nabla_{\max } u\right)$ and

$$
\nabla_{\max }|u|=\operatorname{Re}\left(\bar{s} \nabla_{\max } u\right) .
$$

(ii) For all $\varepsilon>0, s_{\varepsilon} \in W_{\mathrm{loc}}^{1,1}(\Lambda)$ with

$$
\nabla_{\max } s_{\varepsilon}=\frac{\nabla_{\max } u-s_{\varepsilon} \nabla_{\max } u_{\varepsilon}}{u_{\varepsilon}} .
$$

(iii) If $\varphi \in W_{\mathrm{loc}}^{1,1}(\Lambda)$ with $|\varphi| \lesssim 1+|u|$, then $s_{\varepsilon} \varphi \in W_{\mathrm{loc}}^{1,1}(\Lambda)$ for all $\varepsilon>0$ and

$$
\nabla_{\max }\left(s_{\varepsilon} \varphi\right)=\varphi \frac{\nabla_{\max } u-s_{\varepsilon} \nabla_{\max } u_{\varepsilon}}{u_{\varepsilon}}+s_{\varepsilon} \nabla_{\max } \varphi
$$

Moreover, assume $A \in L_{\mathrm{loc}}^{2}\left(\Lambda, \mathbb{R}^{d}\right)$ and $u \in H_{A}^{1}(\Lambda)\left(=\mathscr{D}\left(D_{A, \max }\right)\right)$. Then:

(iv) $|u|, u_{\varepsilon} \in H_{A}^{1}(\Lambda)$ for all $\varepsilon>0$ with $\left|\nabla_{\max } u_{\varepsilon}\right| \leqq\left|D_{A, \max } u\right|$ and $\left|\nabla_{\max }\right| u|| \leqq\left|D_{A, \max } u\right|$.

(v) If $\varphi \in H_{A}^{1}(\Lambda)$ with $|\varphi| \lesssim 1+|u|$, we have $s_{\varepsilon} \varphi \in H_{A}^{1}(\Lambda)$ for all $\varepsilon>0$ and

$$
D_{A, \max }\left(s_{\varepsilon} \varphi\right)=\varphi \frac{D_{A, \max } u-s_{\varepsilon} \nabla_{\max } u_{\varepsilon}}{u_{\varepsilon}}+s_{\varepsilon} \nabla_{\max } \varphi .
$$

Proof. For simplicity, we will again write $\nabla$ for $\nabla_{\max }$ and $D_{A}$ for $D_{A, \max }$. The basic strategy of the proof of (i) is well-known, see [22], [40]. The map $\mathbb{R}^{2} \ni(s, t) \rightarrow \sqrt{s^{2}+t^{2}+\varepsilon^{2}}$ is differentiable and the chain rule for Sobolev spaces, [17], Lemma 7.5, shows that $u_{\varepsilon} \in W_{\text {loc }}^{1,1}(\Lambda)$. Using the same reduction argument as in the proof of Lemma 2.3(ii), we can assume that $u$ is bounded. Then $\bar{u} \nabla_{\max } u \in L_{\text {loc }}^{1}(\Lambda)$, and since $u_{\varepsilon}^{2}=\bar{u} u+\varepsilon^{2}$, Lemma 2.3(i) implies that $u_{\varepsilon}^{2} \in W_{\text {loc }}^{1,1}(\Lambda)$ with $\nabla u_{\varepsilon}^{2}=2 u_{\varepsilon} \nabla u_{\varepsilon}=2 \operatorname{Re}(\bar{u} \nabla u)$. Hence $\nabla u_{\varepsilon}=\operatorname{Re}\left(\overline{s_{\varepsilon}} \nabla u\right)$ for all $\varepsilon>0$. The result for $|u|$ follows by taking the limit $\varepsilon \rightarrow 0$, using the definition of the weak derivative and dominated convergence.

Once part (i) of the lemma holds, (iv) is an immediate consequence, since $H_{A}^{1}(\Lambda) \subset W_{\text {loc }}^{1,1}(\Lambda)$ and $\operatorname{Re}\left(\overline{s_{\varepsilon}} D_{A} u\right)=\operatorname{Re}\left(\overline{s_{\varepsilon}} \nabla u\right)$ for all $\varepsilon>0$. 
By Lemma 2.3(ii) and part (i) above, we have $\frac{1}{u_{\varepsilon}} \in W_{\text {loc }}^{1,1}(\Lambda)$. Since $u \frac{\nabla u_{\varepsilon}}{u_{\varepsilon}^{2}}$ and $\frac{\nabla u}{u_{\varepsilon}}$ are locally integrable, Lemma 2.3(i) shows $s_{\varepsilon}=u / u_{\varepsilon} \in W_{\text {loc }}^{1,1}(\Lambda)$ and

$$
\nabla s_{\varepsilon}=\nabla\left(u \frac{1}{u_{\varepsilon}}\right)=\frac{\nabla u}{u_{\varepsilon}}-u \frac{\nabla u_{\varepsilon}}{u_{\varepsilon}^{2}}=\frac{\nabla u-s_{\varepsilon} \nabla u_{\varepsilon}}{u_{\varepsilon}}
$$

which proves (ii).

(iii) Since $s_{\varepsilon}$ is bounded, $s_{\varepsilon} \nabla \varphi \in L_{\text {loc }}^{1}(\Lambda)$. By assumption, $\varphi / u_{\varepsilon}$ is also bounded. Hence, with part (ii), $\varphi \nabla s_{\varepsilon} \in L_{\text {loc }}^{1}(\Lambda)$ and consequently, using Lemma 2.3(i) again, we see that $s_{\varepsilon} \varphi \in W_{\text {loc }}^{1,1}(\Lambda)$ with

$$
\nabla\left(s_{\varepsilon} \varphi\right)=\varphi \frac{\nabla u-s_{\varepsilon} \nabla u_{\varepsilon}}{u_{\varepsilon}}+s_{\varepsilon} \nabla \varphi
$$

Part (v) follows from this, by adding $-i \frac{\varphi}{u_{\varepsilon}} A u$ to the above equation.

\section{The diamagnetic inequalities}

Lemma 3.1. Let $A \in L_{\text {loc }}^{2}\left(\Lambda, \mathbb{R}^{d}\right)$ and $\varphi \geqq 0, \varphi \in \mathscr{D}\left(\nabla_{\max }\right), \quad u \in \mathscr{D}\left(D_{A, \max }\right)$ with $\varphi \lesssim 1+|u|$. Then $s_{\varepsilon} \varphi \in \mathscr{D}\left(D_{A, \max }\right)$ and the pointwise inequality

$$
\operatorname{Re}\left(\overline{D_{A, \max }\left(s_{\varepsilon} \varphi\right)} \cdot D_{A, \max }(u)\right) \geqq\left|s_{\varepsilon}\right| \nabla_{\max } \varphi \cdot \nabla_{\max }|u|
$$

holds for all $\varepsilon>0$.

Remark 3.2. A similar inequality to the one given in Lemma 3.1, more precisely its $\varepsilon=0$ limit, has already been used in [27], proof of Lemma 6 , for $\Lambda=\mathbb{R}^{d}$ and in [13] to prove a diamagnetic inequality for the Dirichlet operator on open subsets $\Lambda \subset \mathbb{R}^{d}$. There it is enough to consider $\varphi, u \in \mathscr{C}_{0}^{\infty}$, which simplifies some calculations.

Proof. That $s_{\varepsilon} \varphi \in \mathscr{D}\left(D_{A, \max }\right)$ follows from Lemma 2.5(iv). We claim

$$
\operatorname{Re}\left(\overline{D_{A, \max }\left(s_{\varepsilon} \varphi\right)} \cdot D_{A, \max }(u)\right)=\varphi \frac{\left|D_{A, \max } u\right|^{2}-\left|\nabla_{\max } u_{\varepsilon}\right|^{2}}{u_{\varepsilon}}+\left|s_{\varepsilon}\right| \nabla_{\max } \varphi \cdot \nabla_{\max }|u|
$$

from which the inequality in the lemma follows, since $\varphi \geqq 0$ and $\left|\nabla_{\max } u_{\varepsilon}\right| \leqq\left|D_{A, \max } u\right|$ by Lemma 2.5(iv). Using Lemma 2.5(v) gives

$$
\begin{aligned}
\operatorname{Re}\left(\overline{D_{A, \max }\left(s_{\varepsilon} \varphi\right)} \cdot D_{A, \max }(u)\right) & =\varphi \frac{\left|D_{A, \max } u\right|^{2}-\nabla_{\max } u_{\varepsilon} \operatorname{Re}\left(\overline{s_{\varepsilon}} D_{A, \max } u\right)}{u_{\varepsilon}}+\nabla_{\max } \varphi \operatorname{Re}\left(\overline{s_{\varepsilon}} D_{A, \max } u\right) \\
& =\varphi \frac{\left|D_{A, \max } u\right|^{2}-\left|\nabla_{\max } u_{\varepsilon}\right|^{2}}{u_{\varepsilon}}+\nabla_{\max } \varphi \nabla_{\max } u_{\varepsilon}
\end{aligned}
$$


where we also used Lemma 2.5(i). From the above equality, (3.1) follows, since

$$
\nabla_{\max } u_{\varepsilon}=\frac{1}{u_{\varepsilon}} \nabla_{\max } u_{\varepsilon}^{2}=\frac{1}{u_{\varepsilon}} \nabla_{\max }|u|^{2}=\frac{|u|}{u_{\varepsilon}} \nabla_{\max }|u|=\left|s_{\varepsilon}\right| \nabla|u|
$$

Theorem 3.3 (Slight generalization of Theorem 1.1). Let $\Lambda \subset \mathbb{R}^{d}$ be open, $A \in L_{\mathrm{loc}}^{2}\left(\Lambda, \mathbb{R}^{d}\right)$, and $0 \leqq V \in L_{\mathrm{loc}}^{1}(\Lambda)$. Then, for all $t \geqq 0$ and $f \in L^{2}(\Lambda)$,

$$
\left|\mathrm{e}^{-t H_{\Lambda}^{N}(A, V)} f\right| \leqq \mathrm{e}^{-t H_{\Lambda}^{N}(0, V)}|f|
$$

and

$$
\left|\mathrm{e}^{-t H_{\Lambda}^{N}(0, V)} f\right| \leqq \mathrm{e}^{-t H_{\Lambda}^{N}(0,0)}|f|
$$

Moreover, if $V=V_{+}-V_{-}$with $V_{ \pm} \geqq 0$ and $V_{+} \in L_{\text {loc }}^{1}(\Lambda)$, then $V_{-}$relatively form bounded w.r.t. $H_{\Lambda}^{N}(0,0)$ with relative bound $a<1$ implies that $V_{-}$is relatively form bounded w.r.t. $H_{\Lambda}^{N}(A, 0)$ with relative bound $\tilde{a} \leqq a<1$. Moreover, the two bounds

$$
\left|\mathrm{e}^{-t H_{\Lambda}^{N}(A, V)} f\right| \leqq \mathrm{e}^{-t H_{\Lambda}^{N}(0, V)}|f|
$$

and

$$
\left|\mathrm{e}^{-t H_{\Lambda}^{N}(0, V)} f\right| \leqq \mathrm{e}^{-t H_{\Lambda}^{N}(0, \tilde{V})}|f|
$$

hold for all $f \in L^{2}(\Lambda)$ and all potentials $\tilde{V} \leqq V$ for which $\tilde{V}_{-}$is form small with respect to $H_{\Lambda}^{N}(0,0)$.

Remarks 3.4. (i) The same result holds for the Dirichlet semigroup, that is, $\left|\mathrm{e}^{-t H_{\Lambda}^{D}(A, V)} f\right| \leqq \mathrm{e}^{-t H_{\Lambda}^{D}(0, V)}|f| \leqq \mathrm{e}^{-t H_{\Lambda}^{D}(0, \tilde{V})}|f|$. For example, see [6] where this is proven under slightly more restrictive conditions on the vector potential $A$ using the FeynmanKac-Itô formula. With (3.11) below, the Dirichlet case is an immediate corollary of the result for Neumann boundary conditions.

(ii) What we call diamagnetic inequality is often rephrased by semigroup people saying the magnetic semigroup is dominated by the non-magnetic one. In this direction one can find, e.g., in [29], [30], more general (as far as the singularities of $V$ and $A$ are concerned) diamagnetic inequalities for the Dirichlet semigroup.

(iii) The other proofs of the diamagnetic inequality mainly use the $\varepsilon=0$ limit of Lemma 3.1 or similar bounds; see [27], [13], [29], [34]. This forces one to assume $\varphi \lesssim|u|$. We deviate from this line of reasoning by trying to take the limit $\varepsilon \rightarrow 0$ as late as possible in the proof.

Proof. We start with the following well-known remark: Let $A, B$ be self-adjoint operators which are bounded from below. Then the bounds

$$
\left|\mathrm{e}^{-t B} f\right| \leqq \mathrm{e}^{-t A}|f|
$$


for all $f$ and $t \geqq 0$, and

$$
\left|(B+E)^{-n} f\right| \leqq(A+E)^{-n}|f|
$$

for all $f, n \in \mathbb{N}$, and $E>0$ large enough, are equivalent. This follows from the representations

$$
\mathrm{e}^{-t B}=\underset{n \rightarrow \infty}{\mathrm{s}-\lim _{n \rightarrow \infty}}(n / t)(B+n / t)^{-n}
$$

and

$$
(B+E)^{-\alpha}=c_{\alpha} \int_{0}^{\infty} t^{\alpha-1} \mathrm{e}^{-t(B+E)} d t
$$

with $c_{\alpha}^{-1}=\int_{0}^{\infty} t^{\alpha-1} \mathrm{e}^{-t} d t$. In particular, once (3.3) holds for $n \in \mathbb{N}$, it holds for all $n \in(0, \infty)$. As a final preliminary, we note that for $V=V_{+}-V_{-}$with $0 \leqq V_{+} \in L_{\text {loc }}^{1}$ and $V_{-}$form small with respect to $h_{\Lambda}^{N}\left(0, V_{+}\right)$, the first Beurling-Deny criterion implies that the resolvent $\left(H_{\Lambda}^{N}(0, V)+E\right)^{-1}$ is positivity preserving for large enough $E>0$. Similarly, the second Beurling-Deny criterion shows that, for non-negative $V \in L_{\text {loc }}^{1}(\Lambda),\left(H_{\Lambda}^{N}(0, V)+E\right)^{-1}$ maps bounded functions to bounded functions with $\left\|\left(H_{\Lambda}^{N}(0, V)+E\right)^{-1} f\right\|_{\infty} \leqq \frac{1}{E}\|f\|_{\infty}$; see, e.g., [41], Theorems XIII.50, XIII.51, and problem 99. If $V$ is merely bounded from below, the analogous result holds for $E>-$ inf $V$.

In the following we will always assume that $A \in L_{\text {loc }}^{2}\left(\Lambda, \mathbb{R}^{d}\right)$. For the moment, we also assume that $V=0$. Note that $\bar{s}_{\varepsilon} u=\left|s_{\varepsilon}\right||u|$. Let $E>0$. With Lemma 3.1, we get

$$
\begin{aligned}
\left\langle\left|s_{\varepsilon}\right| \nabla_{\max } \varphi, \nabla_{\max }|u|\right\rangle+E\left\langle\left|s_{\varepsilon}\right| \varphi, u\right\rangle & \leqq \operatorname{Re}\left(\left(h_{\Lambda}^{N}[A, 0]+E\right)\left[s_{\varepsilon} \varphi, u\right]\right) \\
& \leqq\left|\left(h_{\Lambda}^{N}[A, 0]+E\right)\left[s_{\varepsilon} \varphi, u\right]\right|
\end{aligned}
$$

for any bounded, non-negative $\varphi \in \mathscr{D}\left(\nabla_{\max }\right)$ and all $\varepsilon>0$. Now write

$$
u=\left(H_{\Lambda}^{N}(A, 0)+E\right)^{-1} f \text { for some } f \in L^{2}(\Lambda) .
$$

Then (3.5) becomes

$$
\left\langle\left|s_{\varepsilon}\right| \nabla_{\max } \varphi, \nabla_{\max }|u|\right\rangle+E\left\langle\left|s_{\varepsilon}\right| \varphi, u\right\rangle \leqq\left|\left\langle s_{\varepsilon} \varphi, f\right\rangle\right| \leqq\langle\varphi,|f|\rangle
$$

Since $\nabla|u|=0$ on $\{u=0\}$ by Lemma $2.5(\mathrm{i})$, we have that $\left|s_{\varepsilon}\right| \nabla|u|$ tends to $\nabla|u|$ in $L^{2}(\Lambda)$ as $\varepsilon \rightarrow 0$. So taking $\varepsilon \rightarrow 0$ in $(3.6)$ gives

$$
\left(h_{\Lambda}^{N}(0,0)+E\right)[\varphi,|u|] \leqq\langle\varphi,|f|\rangle .
$$

Setting $\varphi=\left(H_{\Lambda}^{N}(0,0)+E\right)^{-1} \psi$ for $\psi \in L^{2}(\Lambda)$ non-negative and bounded preserves nonnegativity and boundedness of $\varphi$ by the second remark at the beginning of the proof. Thus (3.7) gives 


$$
\begin{aligned}
\left\langle\psi,\left|\left(H_{\Lambda}^{N}(A, 0)+E\right)^{-1} f\right|\right\rangle & \leqq\left\langle\left(H_{\Lambda}^{N}(0,0)+E\right)^{-1} \psi,|f|\right\rangle \\
& =\left\langle\psi,\left(H_{\Lambda}^{N}(0,0)+E\right)^{-1}|f|\right\rangle
\end{aligned}
$$

for all bounded $0 \leqq \psi \in L^{2}(\Lambda)$ and all $f \in L^{2}(\Lambda)$, which is equivalent to

$$
\left|\left(H_{\Lambda}^{N}(A, 0)+E\right)^{-1} f\right| \leqq\left(H_{\Lambda}^{N}(0,0)+E\right)^{-1}|f|
$$

for all $E>0$. Iterating the last inequality, we get

$$
\left|\left(H_{\Lambda}^{N}(A, 0)+E\right)^{-n} f\right| \leqq\left(H_{\Lambda}^{N}(0,0)+E\right)^{-n}|f|
$$

for all $n \in \mathbb{N}$, which is the diamagnetic inequality for resolvents. By the first remark at the beginning of the proof, the inequality

$$
\left|\mathrm{e}^{-t H_{\Lambda}^{N}(A, 0)} f\right| \leqq \mathrm{e}^{-t H_{\Lambda}^{N}(0,0)}|f| \quad \text { for all } t \geqq 0, f \in L^{2}(\Lambda)
$$

follows.

Now let $V \geqq 0$ and add $\left\langle s_{\varepsilon} \varphi, V u\right\rangle=\left\langle\left|s_{\varepsilon}\right| \varphi, V|u|\right\rangle \geqq 0$ to both sides of (3.4). Setting $u=\left(H_{\Lambda}^{N}(A, V)+E\right)^{-1} f$, we get

$$
\begin{aligned}
\left\langle\left|s_{\varepsilon}\right| \nabla_{\max } \varphi, \nabla_{\max }|u|\right\rangle+\left\langle\left|s_{\varepsilon}\right| \varphi,(V+E) u\right\rangle & \leqq\left|\left(h_{\Lambda}^{N}[A, V]+E\right)\left[s_{\varepsilon}, u\right]\right| \\
& \leqq\left|\left\langle s_{\varepsilon} \varphi, f\right\rangle\right| \leqq\langle\varphi,|f|\rangle
\end{aligned}
$$

for all $0 \leqq \varphi \in \mathscr{D}(\nabla) \cap \mathscr{Q}(V)$. Again, if $\psi$ is non-negative and bounded, so is $\varphi=\left(H_{\Lambda}^{N}(0, V)+E\right)^{-1} \psi$. In turn, we can do the limit $\varepsilon \rightarrow 0$ to get

$$
\left\langle\psi,\left|\left(H_{\Lambda}^{N}(A, V)+E\right)^{-1} f\right|\right\rangle \leqq\left\langle\psi,\left(H_{\Lambda}^{N}(0, V)+E\right)^{-1}|f|\right\rangle
$$

which leads to

$$
\left|\left(H_{\Lambda}^{N}(A, V)+E\right)^{-n} f\right| \leqq\left(H_{\Lambda}^{N}(0, V)+E\right)^{-n}|f|
$$

for all $n \in \mathbb{N}$ and $f \in L^{2}(\Lambda)$, and

$$
\left|\mathrm{e}^{-t H_{\Lambda}^{N}(A, V)} f\right| \leqq \mathrm{e}^{-t H_{\Lambda}^{N}(0, V)}|f| \quad \text { for all } t \geqq 0, f \in L^{2}(\Lambda) .
$$

Now consider the special case $A=0$ and add $\left\langle s_{\varepsilon} \varphi, V u\right\rangle=\left\langle\left|s_{\varepsilon}\right| \varphi, V|u|\right\rangle \geqq 0$ only to the right-hand side of (3.4). Making the obvious changes in the above argument leads to the bound

$$
\left|\left(H_{\Lambda}^{N}(0, V)+E\right)^{-n} f\right| \leqq\left(H_{\Lambda}^{N}(0,0)+E\right)^{-n}|f|
$$

for all $n \in \mathbb{N}$, or equivalently,

$$
\left|\mathrm{e}^{-t H_{\Lambda}^{N}(0, V)} f\right| \leqq \mathrm{e}^{-t H_{\Lambda}^{N}(0,0)}|f|
$$


for all $f \in L^{2}(\Lambda)$. Combining this with (3.9) proves

$$
\left|\mathrm{e}^{-t H_{\Lambda}^{N}(A, V)} f\right| \leqq \mathrm{e}^{-t H_{\Lambda}^{N}(0,0)}|f|
$$

or equivalently,

$$
\left|\left(H_{\Lambda}^{N}(A, V)+E\right)^{-\alpha} f\right| \leqq\left(H_{\Lambda}^{N}(0,0)+E\right)^{-\alpha}|f|
$$

for all $\alpha>0$, non-negative $V \in L_{\text {loc }}^{1}(\Lambda)$, and $f \in L^{2}(\Lambda)$. This proves the first and second inequalities in the theorem. Of course, by adding a constant, one sees that the results remain true if $V$ is merely bounded from below.

The fact that $V_{-}$relatively $H_{\Lambda}^{N}(0,0)$-form bounded with bound $a$ implies that it is relatively $H_{\lambda}^{n}(A, 0)$-form bounded with bound $\tilde{a} \leqq a$ follows immediately from the diamagnetic inequality for the resolvent and the formula

$$
a=\lim _{E \rightarrow \infty}\left\|\left(H_{\Lambda}^{N}+E\right)^{-1 / 2} V_{-}\left(H_{\Lambda}^{N}+E\right)^{-1 / 2}\right\|
$$

for the relative form bound $a$, see, e.g., [9].

Now assume that $V=V_{+}-V_{-}$with $V_{-}$not necessarily bounded but form small with respect to $H_{\Lambda}^{N}(A, 0)$ and the same for $\tilde{V} \leqq V$. For $n \in \mathbb{N}$ set $V_{n}=\max (V,-n)$ and $\tilde{V}_{n}=\max (\tilde{V},-n)$. Using what we proved so far, we know that

$$
\left|\mathrm{e}^{-t H_{\Lambda}^{N}\left(A, V_{n}\right)} f\right| \leqq \mathrm{e}^{-t H_{\Lambda}^{N}\left(0, V_{n}\right)}|f| .
$$

Furthermore, we have

$$
\mathrm{e}^{-t H_{\Lambda}^{N}\left(0, V_{n}\right)}|f| \leqq \mathrm{e}^{-t H_{\Lambda}^{N}\left(0, \tilde{V}_{n}\right)}|f|
$$

which follows similarly to the reasoning in the proof of (3.9) by adding $V_{n}$ to the right and $\tilde{V}_{n}$ to the left-hand side of (3.4) using $\tilde{V}_{n} \leqq V_{n}$ for all $n \in \mathbb{N}$. Letting $n \rightarrow \infty$, the strong convergence guaranteed, for example, by Theorem A.1, finishes the proof of the theorem.

The next simple lemma is our main observation in extending the usual diamagnetic inequality to an inequality for the semigroup differences.

Lemma 3.5. Let $V=V_{+}-V_{-}$with $V_{+} \in L_{\mathrm{loc}}^{1}(\Lambda), V_{-}$form small with respect to $H_{\Lambda}^{N}(0,0)$ and $U$ be any non-negative bounded function. Then

$$
\left|\left(\mathrm{e}^{-t H_{\Lambda}^{N}(A, V)}-\mathrm{e}^{-t H_{\Lambda}^{N}(A, V+U)}\right) f\right| \leqq\left(\mathrm{e}^{-t H_{\Lambda}^{N}(0, V)}-\mathrm{e}^{-t H_{\Lambda}^{N}(0, V+U)}\right)|f|
$$

for all $f \in L^{2}(\Lambda)$. Furthermore, if $\tilde{V} \leqq V$ is such that $\tilde{V}_{-}$is form small with respect to $H_{\Lambda}^{N}(0,0)$, then

$$
\left|\left(\mathrm{e}^{-t H_{\Lambda}^{N}(0, V)}-\mathrm{e}^{-t H_{\Lambda}^{N}(0, V+U)}\right) f\right| \leqq\left(\mathrm{e}^{-t H_{\Lambda}^{N}(0, \tilde{V})}-\mathrm{e}^{-t H_{\Lambda}^{N}(0, \tilde{V}+U)}\right)|f|
$$

for all $f \in L^{2}(\Lambda)$. 
Proof. Let $C, D$ be non-negative operators with $D-C$ bounded. Then

$$
\begin{aligned}
\mathrm{e}^{-t C}-\mathrm{e}^{-t D} & =\int_{0}^{t} \frac{d}{d s}\left(\mathrm{e}^{-s C} \mathrm{e}^{-(t-s) D}\right) d s \\
& =\int_{0}^{t} \mathrm{e}^{-s C}(D-C) \mathrm{e}^{-(t-s) D} d s
\end{aligned}
$$

which is Duhamel's formula. With $D=H_{\Lambda}^{N}(A, V)$ and $C=H_{\Lambda}^{N}(A, V)+U$, we get, for all $f \in L^{2}(\Lambda)$,

$$
\begin{aligned}
\left|\left(\mathrm{e}^{-t H_{\Lambda}^{N}(A, V)}-\mathrm{e}^{-t H_{\Lambda}^{N}(A, V+U)}\right) f\right| & \leqq \int_{0}^{t}\left|\mathrm{e}^{-s H_{\Lambda}^{N}(A, V)} U \mathrm{e}^{-(t-s) H_{\Lambda}^{N}(A, V+U)} f\right| d s \\
& \leqq \int_{0}^{t} \mathrm{e}^{-s H_{\Lambda}^{N}(0, V)} U \mathrm{e}^{-(t-s) H_{\Lambda}^{N}(0, V+U)}|f| d s \\
& =\left(\mathrm{e}^{-t H_{\Lambda}^{N}(0, V)}-\mathrm{e}^{-t H_{\Lambda}^{N}(0, V+U)}\right)|f|
\end{aligned}
$$

by the triangle inequality, a repeated use of the diamagnetic inequality for $\mathrm{e}^{-t H_{\Lambda}^{N}(A, V)}$ and $\mathrm{e}^{-t H_{\Lambda}^{N}(A, V+U)}$, the positivity of $U$, and Duhamel's formula. This proves the first inequality. The second one follows along the same line, using again Duhamel's formula and the bound $\left|\mathrm{e}^{-t H_{\Lambda}^{N}(0, V)} f\right| \leqq \mathrm{e}^{-t H_{\Lambda}^{N}(0, \tilde{V})}|f|$ from Theorem 3.3.

Remark 3.6. Alternatively, one can use the Trotter product formula in place of Duhamel's formula. A third possibility is to use resolvent methods directly: by the remark in the beginning of the proof of Theorem 3.3, the bound in Lemma 3.5 is equivalent to

$$
\begin{aligned}
& \left|\left(\left(H_{\Lambda}^{N}(A, V)+E\right)^{-n}-\left(H_{\Lambda}^{N}(A, V+U)+E\right)^{-n}\right) f\right| \\
& \quad \leqq\left(\left(H_{\Lambda}^{N}(0, V)+E\right)^{-n}-\left(H_{\Lambda}^{N}(0, V+U)+E\right)^{-n}\right)|f| \\
& \quad \leqq\left(\left(H_{\Lambda}^{N}(0, \tilde{V})+E\right)^{-n}-\left(H_{\Lambda}^{N}(0, \tilde{V}+U)+E\right)^{-n}\right)|f|
\end{aligned}
$$

for all $E>-\inf \sigma\left(H_{\Lambda}^{N}(0, \tilde{V})\right)$ and $n \in \mathbb{N}$. For $n=1$, the bound (3.10) can be seen by inspection, using the resolvent identity and the diamagnetic inequality for the resolvents. The case $n \geqq 2$ then follows from the $n=1$ case by writing the difference as a telescoping sum, using the triangle inequality, the bound for $n=1$, and the diamagnetic inequality in each term of the sum.

With this we can finally prove our main result.

Theorem 3.7 (Slight generalization of Theorem 1.3). Let $\Lambda \subset \mathbb{R}^{d}$ be open, $A \in L_{\mathrm{loc}}^{2}\left(\Lambda, \mathbb{R}^{d}\right)$, and $V=V_{+}-V_{-}$with $V_{ \pm} \geqq 0$ and $V_{+} \in L_{\mathrm{loc}}^{1}(\Lambda), V_{-}$relatively form bounded w.r.t. $H_{\Lambda}^{N}(0,0)$. Then

$$
\left|\left(\mathrm{e}^{-t H_{\Lambda}^{N}(A, V)}-\mathrm{e}^{-t H_{\Lambda}^{D}(A, V)}\right) f\right| \leqq\left(\mathrm{e}^{-t H_{\Lambda}^{N}(0, V)}-\mathrm{e}^{-t H_{\Lambda}^{D}(0, V)}\right)|f|
$$

for all $f \in L^{2}(\Lambda)$. Furthermore, if $\tilde{V} \leqq V$ is such that $\tilde{V}_{-}$is form small with respect to $H_{\Lambda}^{N}(0,0)$ then 


$$
\left|\left(\mathrm{e}^{-t H_{\Lambda}^{N}(0, V)}-\mathrm{e}^{-t H_{\Lambda}^{D}(0, V)}\right) f\right| \leqq\left(\mathrm{e}^{-t H_{\Lambda}^{N}(0, \tilde{V})}-\mathrm{e}^{-t H_{\Lambda}^{D}(0, \tilde{V})}\right)|f|
$$

for all $f \in L^{2}(\Lambda)$.

Proof. Given Lemma 3.5, the proof of this theorem reduces to the existence of a non-negative function $U \in L_{\text {loc }}^{1}(\Lambda)$ such that, setting $U_{n}:=\inf (U, n)$,

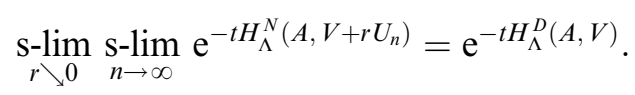

Let $\left\{\Lambda_{n}\right\}_{n \in \mathbb{N}}$ be a sequence of open sets $\Lambda_{n} \subset \Lambda$ such that the closures $\bar{\Lambda}_{n}$ are compact sets, $\bar{\Lambda}_{n} \subset \Lambda_{n+1}$ and $\Lambda=\bigcup_{n} \Lambda_{n}$. Choose smooth functions $0 \leqq \psi_{n} \leqq 1$ with $\psi_{n}(x)=1$ for $x \in \Lambda_{n}$, $\psi_{n}(x)=0$ for $x \in \Lambda_{n+1}$, and set

$$
U(x):=\sum_{n \in \mathbb{N}}\left|\nabla \psi_{n}(x)\right|^{2}
$$

For each $x \in \Lambda$, only one term in the above sum can contribute, so, in fact, $U \in L_{\mathrm{loc}}^{\infty}(\Lambda)$. We claim that $U$ lets us do (3.11). To see this, it is enough to show that

$$
\mathrm{s}-\lim _{n \rightarrow \infty} \mathrm{e}^{-t H_{\Lambda}^{N}\left(A, V+r U_{n}\right)}=\mathrm{e}^{-t H_{\Lambda}^{N}(A, V+r U)}
$$

for fixed $r>0$ and

$$
\mathrm{s}-\lim _{r \searrow 0} \mathrm{e}^{-t H_{\Lambda}^{N}(A, V+r U)}=\mathrm{e}^{-t H_{\Lambda}^{D}(A, V)},
$$

the second being the slightly trickier task. Let $h_{\Lambda}^{N}(A, V)$ be the quadratic form corresponding to $H_{\Lambda}^{N}(A, V)$. Then for any non-negative, locally integrable potential $U$, $H_{\Lambda}^{N}(A, V+U)$ is defined as the operator corresponding to the closed quadratic form

$$
h_{\Lambda}^{N}(A, V+U)[\varphi, \varphi]=h_{\Lambda}^{N}(A, V)[\varphi, \varphi]+\langle\sqrt{U} \varphi, \sqrt{U} \varphi\rangle
$$

with domain $\mathscr{D}\left(h_{\Lambda}^{N}(A, V+U)\right)=\mathscr{D}\left(h_{\Lambda}^{N}(A, V)\right) \cap \mathscr{D}(\sqrt{U})$.

Since $U_{n}$ converges pointwise monotonically to $U_{n}$, we can use Lebesgue's monotone convergence theorem to see that $h_{\Lambda}^{N}\left(A, V+r U_{n}\right)$ converges monotonically to $h_{\Lambda}^{N}(A, V+r U)$. By Theorem A.1(i), the corresponding operators converge in strong resolvent sense. In particular, for any fixed $r>0$, (3.13) holds.

The proof of (3.14) is more complicated: Since

$$
\bigcup_{r>0} \mathscr{D}\left(h_{\Lambda}^{N}(A, V+r U)\right)=h_{\Lambda}^{N}(A, V+U)=\mathscr{D}\left(h_{\Lambda}^{N}(A, V)\right) \cap \mathscr{D}(\sqrt{U}),
$$

we see that $\lim _{r \rightarrow 0} h_{\Lambda}^{N}(A, V+r U)[\varphi, \varphi]=h_{\Lambda}^{N}(A, V)[\varphi, \varphi]$ for all $\varphi \in \bigcup_{r>0} \mathscr{D}\left(h_{\Lambda}^{N}(A, V+r U)\right)$. In order to apply Theorem A.1(ii), it remains to show that

$$
\overline{\mathscr{D}\left(h_{\Lambda}^{N}(A, V+U)\right)} \|^{\|\cdot\|_{\Lambda}^{D}(A, V)}=\mathscr{D}\left(h_{\Lambda}^{D}(A, V)\right)=H_{0, A}^{1}(\Lambda) \cap \mathscr{Q}(V) .
$$


Since $\mathscr{C}_{0}^{\infty}(\Lambda)$ is dense in $\mathscr{2}\left(V_{+}\right)$with respect to $\|\cdot\|_{V_{+}}$and

$$
\mathscr{D}\left(h_{\Lambda}^{N}(A, V+U)\right)=\mathscr{D}\left(h_{\Lambda}^{N}(A, 0)\right) \cap \mathscr{Z}\left(V_{+}\right) \cap \mathscr{Z}(U) \subset \mathscr{D}\left(h_{\Lambda}^{N}(A, U)\right),
$$

this is the case if

$$
\mathscr{C}_{0}^{\infty}(\Lambda) \text { is dense in } \mathscr{D}\left(h_{\Lambda}^{N}(A, U)\right) \text { in the norm }\|\cdot\|_{h_{\Lambda}^{D}(A, 0)} .
$$

We show (3.15) in three steps:

Step 1: $\mathscr{D}\left(h_{\Lambda}^{N}(A, U)\right) \cap L^{\infty}(\Lambda)$ is dense in $\mathscr{D}\left(h_{\Lambda}^{N}(A, U)\right)$. We follow [27], proof of Lemma 2, quite closely, but with some slight simplifications, since we cut smoothly in the range of functions: Given $n \in \mathbb{N}, n \geqq 2$, let $\varphi_{n} \in \mathscr{C}^{1}([0, \infty))$ with $0 \leqq \varphi_{n} \leqq 1, \varphi_{n}(t)=1$ for all $0 \leqq t \leqq n-1, \varphi_{n}(t)=(n-1 / 2) / t$ for $t \geqq n$ and $\sup ^{\prime}\left|\varphi^{\prime}(t)\right| \leqq\left|\varphi^{\prime}(n)\right| \leqq 1 / n$. For such a sequence of functions, we have the bounds $\left(1-\varphi_{n}(t)\right) \leqq \chi_{[n-1, \infty)}(t)$ and $t\left|\varphi_{n}^{\prime}(t)\right| \leqq \chi_{[n-1, \infty)}(t)$. Given $u \in \mathscr{D}\left(h_{\Lambda}^{N}(A, U)\right)$, set $u_{n}:=\varphi_{n}\left(u_{1}\right) u$, where $u_{1}=\sqrt{|u|^{2}+1}$. Then, as distributions,

$$
\nabla u_{n}=\varphi_{n}\left(u_{1}\right) \nabla u+u \varphi_{n}^{\prime}\left(u_{1}\right) \nabla u_{1}
$$

Hence

$$
D_{A} u-D_{A} u_{n}=\left(1-\varphi_{n}\left(u_{1}\right)\right) D_{A} u-u \varphi_{n}^{\prime}\left(u_{1}\right) \nabla u_{1}
$$

By Lemma 2.5(iv), we know $\nabla u_{1} \in L^{2}(\Lambda)$. Note that $\left|u \varphi_{n}^{\prime}\left(u_{1}\right)\right| \leqq u_{1}\left|\varphi_{n}^{\prime}\left(u_{1}\right)\right| \leqq \chi_{[n-1, \infty)}\left(u_{1}\right)$. Thus, by (3.16), $\left\|D_{A} u-D_{A} u_{n}\right\|$ goes to zero as $n \rightarrow \infty$.

Step 2: $\mathscr{D}\left(h_{\Lambda}^{N}(A, U)\right) \cap L_{\text {comp }}^{\infty}(\Lambda)$ is dense in $\mathscr{D}\left(h_{\Lambda}^{N}(A, U)\right)$. By Step 1 we can assume $u \in \mathscr{D}\left(h_{\Lambda}^{N}(A, U)\right) \cap L_{\text {comp }}^{\infty}(\Lambda)$. Recall that $U=\sum_{n}\left|\nabla \psi_{n}\right|^{2}$. Put $u_{n}:=\psi_{n} u$ and note that $u_{n}$ has compact support in $\Lambda$. As distributions,

$$
D_{A} u-D_{A} u_{n}=\left(1-\psi_{n}\right) D_{A} u-u \nabla \psi_{n}
$$

We have $\sum_{n} u \nabla \psi_{n} \in L^{2}(\Lambda)$ since $u \in \mathscr{Q}(U)$. In particular, $u \nabla \psi_{n}$ goes to zero in $L^{2}(\Lambda)$. Hence, by (3.17), $\left\|D_{A} u-D_{A} u_{n}\right\|$ goes to zero as $n \rightarrow \infty$.

Step 3: $\mathscr{C}_{0}^{\infty}(\Lambda)$ is dense in $\mathscr{D}\left(h_{\Lambda}^{N}(A, U)\right)$. Without loss of generality we can assume $u \in \mathscr{D}\left(h_{\Lambda}^{N}(A, U)\right) \cap L_{\text {comp }}^{\infty}(\Lambda)$. Since $A \in L_{\text {loc }}^{2}\left(\Lambda, \mathbb{R}^{d}\right)$, we know that

$$
A u \in L^{2}(\Lambda) \text { and } \nabla u=D_{A} u+i A u \in L^{2}(\Lambda) .
$$

Let $\left(j_{n}\right)_{n \in \mathbb{N}}$ be a sequence of approximate delta functions with $\operatorname{supp}\left(j_{n}\right) \subset B_{1 / n}(0)$. Choose $n$ so large that $u_{n}:=j_{n} * u \in \mathscr{C}_{0}^{\infty}(\Lambda)$. By standard mollifier arguments, $\left\|\nabla u-\nabla u_{n}\right\|$ and $\left\|A u-A u_{n}\right\|$ go to zero as $n \rightarrow \infty$. Since

$$
D_{A} u-D_{A} u_{n}=\nabla u-\nabla u_{n}-i A u+i A u_{n},
$$

this finishes the proof of $(3.15)$. 
Remark 3.8. The proof of (3.15) is motivated by the proof of the Feynman-Kac formula for the Dirichlet Laplacian on arbitrary open subsets in [48]. That one can extend this line of thought to certain magnetic vector potentials was already noticed in [6]. The observation that the ideas in [48] even allow one to recover Dirichlet from Neumann boundary conditions for arbitrary vector potentials in $L_{\text {loc }}^{2}\left(\Lambda, \mathbb{R}^{d}\right)$ seems to be new.

Proof of Corollary 1.4. Part (i). By Remark 1.5(ii), we know that the free semigroup $\mathrm{e}^{-t H_{\Lambda}^{N}(0,0)}$ is a trace class operator, and standard perturbation arguments show that $\mathrm{e}^{-t H_{\Lambda}^{N}\left(0,-V_{-}\right)}$and $\mathrm{e}^{-t H_{\Lambda}^{N}(0, V)}$ are trace class operators, see, e.g., [41], proof of Theorem XIII.76.

In the following, let $\operatorname{tr}$ stand for the trace on $L^{2}(\Lambda)$ and $\|C\|_{H S}=\operatorname{tr}\left[C^{*} C\right]^{1 / 2}$ be the Hilbert-Schmidt norm. Since $\mathrm{e}^{-t H_{\Lambda}^{N}(A, V)}$ is a non-negative operator, its trace norm is equal to its trace and, by the semigroup property and self-adjointness, we have

$$
\operatorname{tr}\left[\mathrm{e}^{-2 t H_{\Lambda}^{N}(A, V)}\right]=\operatorname{tr}\left[\left(\mathrm{e}^{-t H_{\Lambda}^{N}(A, V)}\right)^{*} \mathrm{e}^{-t H_{\Lambda}^{N}(A, V)}\right]=\left\|\mathrm{e}^{-t H_{\Lambda}^{N}(A, V)}\right\|_{H S}^{2} .
$$

From the diamagnetic inequality and [11], Theorem 2.4.4, we know that $\mathrm{e}^{-t H_{\Lambda}^{N}(A, V)}$ is an integral operator whose kernel obeys the bounds

$$
\left|\mathrm{e}^{-t H_{\Lambda}^{N}(A, V)}(x, y)\right| \leqq \mathrm{e}^{-t H_{\Lambda}^{N}(0, V)}(x, y) \leqq \mathrm{e}^{-t H_{\Lambda}^{N}\left(0,-V_{-}\right)}(x, y) \quad \text { for almost all } x, y \in \Lambda
$$

Thus, using [39], Theorem VI.23, we conclude

$$
\begin{aligned}
\operatorname{tr}\left[\mathrm{e}^{-2 t H_{\Lambda}^{N}(A, V)}\right] & =\left\|\mathrm{e}^{-t H_{\Lambda}^{N}(A, V)}\right\|_{H S}^{2}=\iint_{\Lambda \times \Lambda}\left|\mathrm{e}^{-t H_{\Lambda}^{N}(A, V)}(x, y)\right|^{2} d x d y \\
& \leqq\left\|\mathrm{e}^{-t H_{\Lambda}^{N}(0, V)}\right\|_{H S}^{2}=\operatorname{tr}\left[\mathrm{e}^{-2 t H_{\Lambda}^{N}(0, V)}\right]<\infty
\end{aligned}
$$

by the bound on the integral kernels. In an analogous way, one sees that $\operatorname{tr}\left[\mathrm{e}^{-t H_{\Lambda}^{N}(0, V)}\right] \leqq \operatorname{tr}\left[\mathrm{e}^{-t H_{\Lambda}^{N}\left(0,-V_{-}\right)}\right]$.

Part (ii). For any pair $C, D$ of Hilbert-Schmidt operators on $L^{2}(\Lambda)$, an easy extension of the proof of Theorem VI.23 in [39] shows the formula

$$
\operatorname{tr}[C D]=\iint_{\Lambda \times \Lambda} C(x, y) D(y, x) d x d y
$$

We know, by our assumptions on $\Lambda$ and part (i) of the corollary, that $\mathrm{e}^{-t H_{\Lambda}^{N}(A, V)}-\mathrm{e}^{-t H_{\Lambda}^{D}(A, V)}$ is a trace class operator (both $\mathrm{e}^{-t H_{\Lambda}^{N}(A, V)}$ and $\mathrm{e}^{-t H_{\Lambda}^{D}(A, V)}$ are trace class!) and thus, in particular, Hilbert-Schmidt operators. Moreover, the difference of the kernels obeys, by the diamagnetic inequality for the differences, Theorem 3.7,

$$
\begin{aligned}
\left|\mathrm{e}^{-t H_{\Lambda}^{N}(A, V)}(x, y)-\mathrm{e}^{-t H_{\Lambda}^{D}(A, V)}(x, y)\right| & \leqq \mathrm{e}^{-t H_{\Lambda}^{N}(0, V)}(x, y)-\mathrm{e}^{-t H_{\Lambda}^{D}(0, V)}(x, y) \\
& \leqq \mathrm{e}^{-t H_{\Lambda}^{N}\left(0,-V_{-}\right)}(x, y)-\mathrm{e}^{-t H_{\Lambda}^{D}\left(0,-V_{-}\right)}(x, y)
\end{aligned}
$$

for almost all $x, y \in \Lambda$. Factorizing 


$$
\begin{aligned}
\mathrm{e}^{-2 t H_{\Lambda}^{N}(A, V)}-\mathrm{e}^{-2 t H_{\Lambda}^{D}(A, V)}= & \mathrm{e}^{-t H_{\Lambda}^{N}(A, V)}\left(\mathrm{e}^{-t H_{\Lambda}^{N}(A, V)}-\mathrm{e}^{-t H_{\Lambda}^{D}(A, V)}\right) \\
& +\left(\mathrm{e}^{-t H_{\Lambda}^{N}(A, V)}-\mathrm{e}^{-t H_{\Lambda}^{D}(A, V)}\right) \mathrm{e}^{-t H_{\Lambda}^{D}(A, V)},
\end{aligned}
$$

using (3.18), the diamagnetic inequalities for the respective kernels, and reasoning similarly as in part (i) gives the claim in Corollary 1.4(ii).

Remarks 3.9. (i) Of course, with the obvious notational changes, one can extend the above proof to cover the case $\tilde{V} \leqq V$.

(ii) It might be that, for some cleverly constructed bounded set $\Lambda$, the Neumann semigroup $\mathrm{e}^{-t H_{\Lambda}^{N}(0,0)}$ is compact but not trace class or Hilbert-Schmidt for small times $t$, see Remark 1.5(iii). Nevertheless, the diamagnetic inequality and the Dodds, Fremlin, and Pitt theorem [1], [13], [38] ensures that $\mathrm{e}^{-t H_{\Lambda}^{N}(A, 0)}$ is also compact for arbitrary vector potentials $A \in L_{\mathrm{loc}}^{2}\left(\Lambda . \mathbb{R}^{d}\right)$. See also [26] for a nice extension of this basic compactness criterion.

\section{Appendix. Monotone convergence of forms and strong resolvent convergence}

For the convenience of the reader we present here the basic convergence theorem we need in the proof of Theorem 3.7.

Theorem A.1. Let $\mathscr{H}$ be a Hilbert space, $s_{n}, u_{n}$, and $h$ non-negative, closed quadratic forms, and $S_{n}, U_{n}$, and $H$ be the corresponding self-adjoint operators.

(i) If $u_{n} \leqq u_{n+1} \leqq h$ and $h(\varphi, \varphi)=\sup _{n} u_{n}(\varphi, \varphi)$, that is,

$$
\begin{aligned}
& \left\{\varphi \in \bigcap_{n \in \mathbb{N}} \mathscr{D}\left(u_{n}\right): \sup _{n \in \mathbb{N}} u_{n}(\varphi, \varphi)<\infty\right\}=\mathscr{D}(h), \\
& u_{n}(\varphi, \varphi) \rightarrow h(\varphi, \varphi) \quad \text { as } n \rightarrow \infty, \quad \forall \varphi \in \mathscr{D}(h),
\end{aligned}
$$

then $U_{n} \rightarrow H$ as $n \rightarrow \infty$ in the strong resolvent sense.

(ii) If $s_{n} \geqq s_{n+1} \geqq h$ and

$$
\begin{aligned}
& {\overline{\bigcup_{n \in \mathbb{N}} \mathscr{D}\left(s_{n}\right)}}^{\|\cdot\|_{h}}=\mathscr{D}(h), \\
& s_{n}(\varphi, \varphi) \rightarrow h(\varphi, \varphi) \quad \text { as } n \rightarrow \infty, \quad \forall \varphi \in \bigcup_{n \in \mathbb{N}} \mathscr{D}\left(s_{n}\right),
\end{aligned}
$$

then $S_{n} \rightarrow H$ as $n \rightarrow \infty$ in the strong resolvent sense.

Remarks A.2. (i) As usual, see, e.g., [39], Theorem VIII.20, strong resolvent convergence of $U_{n}$ (resp. $\left.S_{n}\right)$ implies strong convergence of $f\left(U_{n}\right)$ (resp. $\left.f\left(S_{n}\right)\right)$ for any continuous bounded function $f$.

(ii) This theorem is taken from Simon [47], see also [39], Theorems S.14 and S.16, where an even stronger result was proven: The quadratic forms need not be densely defined, as long as one interprets strong resolvent convergence correctly. 
(iii) A partly alternate proof of this result can be found in [59].

(iv) In general, (generalized) strong convergence of operators is equivalent to the so-called $\Gamma$-convergence of the corresponding quadratic forms; see [10].

Acknowledgements. It is a pleasure to thank Alexander Elgart for discussions and Hendrik Vogt for pointing us to [30]. D. H. also thanks Ari Laptev and Kjell-Ove Widman for their hospitality at the Mittag-Leffler institute.

\section{References}

[1] C. D. Aliprantis and O. Burkinshaw, Positive compact operators on Banach lattices, Math. Z. 174 (1980), no. 3, 289-298.

[2] J. Avron, I. Herbst, and B. Simon, Schrödinger operators with magnetic fields, I. General interactions, Duke Math. J. 45 (1978), 847-883.

[3] J. Avron and B. Simon, Almost periodic Schrödinger operators, II. The integrated density of states, Duke Math. J. 50 (1983), 369-391.

[4] O. Bratteli and D. W. Robinson, Operator algebras and quantum statistical mechanics. 2, Equilibrium states, Models in quantum statistical mechanics, Second edition, Texts and Monographs in Physics, SpringerVerlag, Berlin 1997.

[5] K. Broderix, D. Hundertmark, and H. Leschke, Self-averaging, decomposition, and asymptotic properties of the density of states for random Schrödinger operators with constant magnetic field, in: Path integrals from meV to MeV, Tutzing 1993, eds. H. Grabert, A. Inomata, L. S. Schulman, and U. Weiss, World Scientific, Singapore 1993.

[6] K. Broderix, D. Hundertmark, and H. Leschke, Continuity properties of Schrödinger semigroups with magnetic fields, Rev. Math. Phys. 12 (2000), no. 2, 181-225.

[7] R. Carmona and J. Lacroix, Spectral theory of random Schrödinger operators, Probability and its Applications, Birkhäuser, Boston 1990.

[8] J. M. Combes, R. Schrader, and R. Seiler, Classical bounds and limits for energy distributions of Hamilton operators in electromagnetic fields, Ann. Physics 111 (1978), no. 1, 1-18.

[9] H. L. Cycon, R. G. Froese, W. Kirsch, and B. Simon, Schrödinger operators: With application to quantum mechanics and global geometry, Springer, Berlin 1984.

[10] G. Dal Maso, An introduction to Г-convergence, Birkhäuser, Boston 1992.

[11] E. B. Davies, Heat kernels and spectral theory, Cambridge Tracts Math. 92, Cambridge University Press, Cambridge 1990.

[12] E. B. Davies and B. Simon, Spectral properties of the Neumann Laplacian of horns, Geom. Funct. Anal. 2 (1992), 105-117.

[13] P. G. Dodds and D. H. Fremlin, Compact operators in Banach lattices, Israel J. Math. 34 (1979), no. 4, 287-320.

[14] S.-I. Doi, A. Iwatsuka, and T. Mine, The uniqueness of the integrated density of states for the Schrödinger operators with magnetic fields, Math. Z. 237 (2001), no. 2, 335-371.

[15] J. Droese and W. Kirsch, The effect of boundary conditions on the density of states for random Schrödinger operators, Stochastic Process. Appl. 23 (1986), no. 1, 169-175.

[16] M. Fisher, The free energy of a macroscopic system, Arch. Rat. Mech. Anal. 17 (1964), 377-410.

[17] D. Gilbarg and N. Trudinger, Elliptic Partial Differential Equations of Second Order, Classics in Mathematics, Springer, Berlin 1998.

[18] R. Hempel, L. Seco, and B. Simon, The essential spectrum of Neumann Laplacians on some bounded singular domains, J. Funct. Anal. 102 (1991), 448-483.

[19] H. Hess, R. Schrader, and D. A. Uhlenbrock, Domination of semigroups and generalization of Kato's inequality, Duke Math. J. 44 (1977), no. 4, 893-904.

[20] T. Hupfer, H. Leschke, P. Müller, and S. Warzel, The absolute continuity of the integrated density of states for magnetic Schrödinger operators with certain unbounded random potentials, Comm. Math. Phys. 221 (2001), no. 2, 229-254.

[21] T. Hupfer, H. Leschke, P. Müller, and S. Warzel, Existence and uniqueness of the integrated density of states for Schrödinger operators with magnetic fields and unbounded random potentials, Rev. Math. Phys. 13 (2001), no. 12, 1547-1581. 
[22] T. Kato, Schrödinger operators with singular potentials, Proceedings of the International Symposium on Partial Differential Equations and the Geometry of Normed Linear Spaces, Jerusalem 1972, Israel J. Math. 13 (1972), 135-148.

[23] W. Kirsch, Random Schrödinger operators. A course, Schrödinger operators (Sønderborg 1988), Lect. Notes Phys. 345, Springer, Berlin (1989), 264-370.

[24] W. Kirsch and F. Martinelli, On the density of states of Schrödinger operators with a random potential, J. Phys. A 15 (1982), no. 7, 2139-2156.

[25] W. Kirsch and F. Martinelli, On the essential selfadjointness of stochastic Schrödinger operators, Duke Math. J. 50 (1983), no. 4, 1255-1260.

[26] H. Leinfelder, A remark on a paper: "A compactness condition for linear operators of function spaces" (J. Oper. Th. 1 (1979), no. 1, 49-54) by L. D. Pitt, Bayreuther Math. Schr. 11 (1982), 57-66.

[27] H. Leinfelder and C. Simader, Schrödinger operators with singular magnetic vector potentials, Math. Z. 176 (1981), no. 1, 1-19.

[28] I. Lifshits, S. Gredeskul, and L. Pastur, Introduction to the theory of disordered systems, Translated from the Russian by Eugene Yankovsky, Wiley-Interscience Publication, John Wiley \& Sons, New York 1988.

[29] V. Liskevich and A. Manavi, Dominated semigroups with singular complex potentials, J. Funct. Anal. 151 (1997), no. 2, 281-305.

[30] A. Manavi, Zur Störung von dominierten $C_{0}$-Halbgruppen auf Banachfunktionenräumen mit ordnungstetiger Norm und sektoriellen Formen mit singulären komplexen Potentialen, Ph.D. Thesis, Technical University Dresden, 2001 (in German).

[31] H. Matsumoto, On the integrated density of states for the Schrödinger operators with certain random electromagnetic potentials, J. Math. Soc. Japan 45 (1993), no. 2, 197-214.

[32] S. Nakamura, A remark on the Dirichlet-Neumann decoupling and the integrated density of states, J. Funct. Anal. 179 (2001), no. 1, 136-152.

[33] S. Nakao, On the spectral distribution of the Schrödinger operator with random potential, Japan. J. Math. (N.S.) 3 (1977), no. 1, 111-139.

[34] E.-M. Ouhabaz, Invariance of closed convex sets and domination criteria for semigroups, Pot. Anal. 5 (1996), no. 6, 611-625.

[35] L. Pastur, The Schrödinger equation with random potential, Theor. Math. Phys. 6 (1971), 299-306, Russian original: Teoret. Mat. Fiz. 6 (1971), no. 3, 415-424.

[36] L. Pastur, Spectral properties of disordered systems in the one-body approximation, Comm. Math. Phys. 75 (1980), no. 2, 179-196.

[37] L. Pastur and A. Figotin, Spectra of random and almost-periodic operators, Fundamental Principles of Mathematical Sciences, Grundl. Math. Wiss. 297, Springer, Berlin 1992.

[38] L. D. Pitt, A compactness condition for linear operators of function spaces, J. Oper. Th. 1 (1979), no. 1, 49-54.

[39] M. Reed and B. Simon, Methods of modern mathematical physics, I: Functional analysis, Revised and enlarged edition, Academic Press, New York 1980.

[40] M. Reed and B. Simon, Methods of modern mathematical physics, II: Self-adjointness, Academic Press, New York 1975.

[41] M. Reed and B. Simon, Methods of modern mathematical physics, IV: Analysis of operators, Academic Press, New York 1978.

[42] G. Roepstorff, Path integral approach to quantum physics. An introduction, Texts and Monographs in Physics, Springer-Verlag, Berlin 1994.

[43] D. Ruelle, Statistical mechanics. Rigorous results, Reprint of the 1989 edition, World Scientific River Edge, NJ; Imperial College Press, London 1999.

[44] E. M. Stein, Singular integrals and differentiability properties of functions, Princeton University Press, Princeton 1970.

[45] B. Simon, Universal diamagnetism of spinless bose systems, Phys. Rev. Lett. 36 (1976), 1083-1084.

[46] B. Simon, An abstract Kato's inequality for generators of positivity preserving semi-groups, Ind. Math. J. 26 (1977), 1067-1073.

[47] B. Simon, A canonical decomposition for quadratic forms with applications to monotone convergence theorems, J. Funct. Anal. 28 (1978), no. 3, 377-385.

[48] B. Simon, Classical boundary conditions as a technical tool in modern mathematical physics, Adv. Math. 30 (1978), no. 3, 268-281.

[49] B. Simon, Functional integration and quantum physics, Academic Press, New York 1979.

[50] B. Simon, Maximal and minimal Schrödinger forms, J. Oper. Th. 1 (1979), 37-47.

[51] B. Simon, Kato's inequality and the comparison of semi-groups, J. Funct. Anal. 32 (1979), 97-101. 
[52] B. Simon, The Neumann Laplacian of a jelly roll, Proc. Amer. Math. Soc. 114 (1992), 783-785.

[53] P. Stollmann, Admissible and regular potentials for Schrödinger forms, J. Oper. Th. 18 (1987), no. 1, 139-151.

[54] P. Stollmann and J. Voigt, A regular potential which is nowhere in $L_{1}$, Lett. Math. Phys. 9 (1985), no. 3, 227-230.

[55] H. Tanaka, Stochastic differential equations with reflecting boundary condition in convex regions, Hiroshima Math. J. 9 (1979), no. 1, 163-177.

[56] N. Ueki, On spectra of random Schrödinger operators with magnetic fields, Osaka J. Math. 31 (1994), no. 1, $177-187$.

[57] J. Voigt, Absorption semigroups, their generators, and Schrödinger semigroups, J. Funct. Anal. 67 (1986), no. 2, 167-205.

[58] J. Voigt, Absorption semigroups, J. Oper. Th. 20 (1988), no. 1, 117-131.

[59] J. Weidmann, Stetige Abhängigkeit der Eigenwerte und Eigenfunktionen elliptischer Differentialoperatoren vom Gebiet, Continuous dependence of eigenvalues and eigenfunctions of elliptic differential operators on the domain, Math. Scand. 54 (1984), no. 1, 51-69 (in German).

[60] H. Weyl, Das asymptotische Verteilungsgesetz der Eigenwerte linearer partieller Differentialgleichungen (mit einer Anwendung auf die Theorie der Hohlraumstrahlung), Math. Ann. 71 (1912), 441-479 (in German).

[61] R. J. Williams, Reflected Brownian motion: Hunt processes and semimartingale representation, in: Barcelona Seminar on Stochastic Analysis (St. Feliu de Guxols 1991), Progr. Probab. 32, Birkhäuser, Basel (1993), 216221.

[62] R. J. Williams and W. A. Zheng, On reflecting Brownian motion-a weak convergence approach, Ann. Inst. H. Poincaré Probab. Statist. 26 (1990), no. 3, 461-488.

Department of Mathematics, University of Illinois at Urbana-Champaign, 1409 W. Green Street,

Urbana, IL 61801

e-mail: dirk@math.uiuc.edu

Department of Mathematics 253-37, California Institute of Technology, Pasadena, CA 91125

e-mail: bsimon@caltech.edu

Eingegangen 14. Januar 2003, in revidierter Fassung 3. März 2003 